\title{
Development of a 2D Analytical Model for the Electromagnetic Computation of Axial-Field Magnetic Gears
}

\author{
Thierry Lubin, Smail Mezani, and Abderrezak Rezzoug. \\ Université de Lorraine, Groupe de Recherche en Electrotechnique et Electronique de Nancy, GREEN, F-54500 Vandœuvre-lès- \\ Nancy, France
}

\begin{abstract}
This paper describes a two-dimensional analytical model to predict the magnetic field distribution in axial-field magnetic gears by using the sub-domain method. The sub-domain method consists in solving the partial differential equations linked to the Maxwell's equations in each rectangular region (magnets, air-gaps and slots) by the separation of variables method. The proposed model is based on a two-dimensional approximation for the magnetic field distribution (mean radius model) i.e. the problem is solved in 2D Cartesian coordinates. One of the main contributions of the paper concerns the analytic solution of the magnetic field in a slot open on the two sides (space between the ferromagnetic pole-pieces). Moreover, it is shown that the analytical model and the 3D finite elements simulations follow the same trends in the determination of the optimum values for the geometrical parameters. As the analytical model takes less computational time than 3D numerical model, it can be used as an effective tool for the first step of design optimization.
\end{abstract}

Index Terms - Analytical solution, axial flux, magnetic gear, magnetic field, electromagnetic torque, axial force.

\section{INTRODUCTION}

$\mathrm{M}$ AGNETIC gear can be used as power transmission system between an input and output shaft without any mechanical contact. The torque between the two shafts is transferred through magnetic field interaction in the air-gap regions. As the torque could be transmitted across a separation wall, magnetic gears are interesting solutions for isolated systems such as pumps. Moreover, they present many advantages compared to mechanical gearboxes such as low acoustic noise, low vibrations, reduced maintenance (contactless system with no lubrication), and inherent overload protection (pull-out torque) [1]-[8].

In order to analyze the performances of an axial magnetic gear, an accurate knowledge of the magnetic field distribution in the air-gap regions is required. The magnetic field distribution can be evaluated by analytical methods or numerical methods. Numerical methods, like finite element analysis (FEA), present the advantage of taking into account the real geometry of the magnetic gear as well as the magnetic saturation of the iron parts. On the other hand, analytical methods, which are based on some simplifying assumptions (simplified geometry, linear characteristic for the material, simple boundary conditions), generally require much less computational time than FEA and can be effective tools for the first step of design optimization.

Among the analytical methods, the sub-domain method has been extensively developed these last years to study the slotting effect in electrical machine and actuators [9]-[16]. The sub-domain method consists in dividing the whole domain of the studied device into elementary rectangular sub-domains (air-gap, magnets, and slots). The exact solution of the magnetic field in each sub-domain is obtained by solving the partial differential equations (PDE) issued from the Maxwell's

Digital Object Identifier inserted by IEEE equations. The sub-domains are linked by the classical interface conditions for the magnetic field. It has been shown that this method is very attractive in terms of accuracy and computational times. Recently, K.J. Meessen et al. have shown that the sub-domain method can be applied for the analysis of 3-D cylindrical structures with slotting effect [25].

The sub-domain method was also used to predict the magnetic field in actuators with more complex geometry such as coaxial magnetic gear [17], [18] or radial-field PMs motors with semi-closed slots [19], [20]. In these cases, the slots are open on two sides (tooth-tips for PMs motors), hence the problem to solve becomes much more complicated. For axialfield actuators or motors, this difficult problem (slots open on two sides) is rarely treated in the literature [21]. However, this "2-sides" opening part is essential to create the torque in axialfield magnetic gears as shown in Fig. 1 and Fig. 2. Therefore, it is important to predict the magnetic field in the slots with a good accuracy.

In [22], a general 2D formulation using Fourier analysis has been developed to study a wide range of electrical actuators in three different 2D coordinate systems (Cartesian, polar and cylindrical). As indicated in [22], open slots on two sides introduce different spatial frequencies in the field solution in the domains adjacent to these two sides (air-gap). Hence, a special attention must be given to treat this problem via an analytical way (this is the case for the studied magnetic gear as shown in Fig.3). The authors in [22] promote the use of an extra boundary condition by setting the divergence of the magnetic field to zero around each ferromagnetic block (teeth) in order to calculate some Fourier coefficients. It will be shown in this paper that this is not necessary as far as the mathematical problem is well posed.

The purpose of this paper is to present a $2 \mathrm{D}$ analytical model of an axial-field magnetic gear based on the sub-domain method. At our best knowledge, the performances prediction of an axial field magnetic gear has never been done by an analytical way. As indicated previously, a special attention is 
given in this paper to the solution of the magnetic field in a slot open on two sides and situated between two other regions (air-gaps) as shown in Fig. 4. The developed analytical model is then used to analyze the performances (torque and axial force) of the gear. In order to study the limits of the proposed 2-D model, the results are compared with those obtained from 3-D finite element simulations. A parametric study is also presented to show the effectiveness of the proposed model.

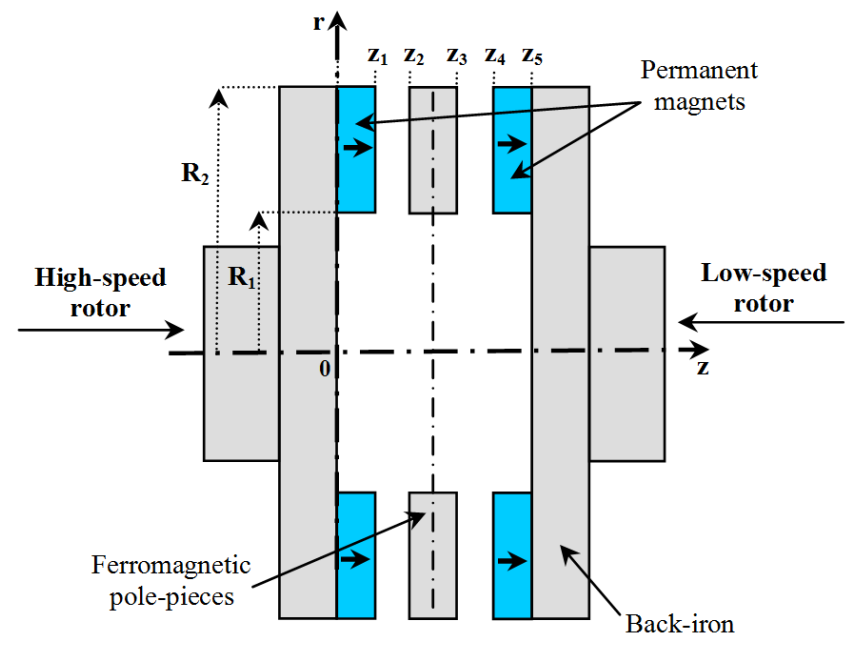

Fig. 1. Geometry of the studied axial-field magnetic gear.

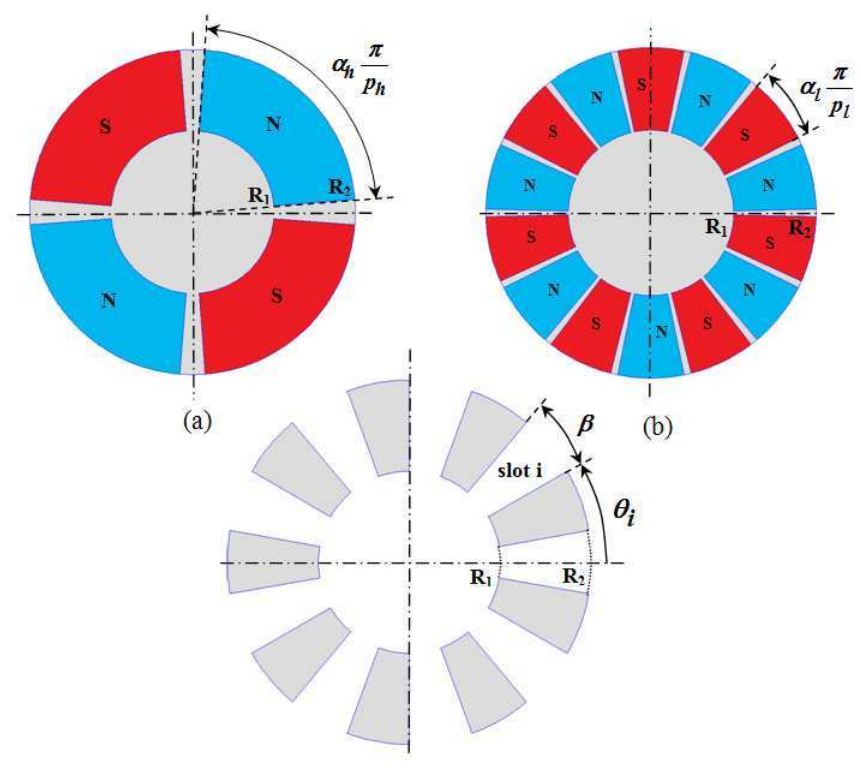

(c)

Fig. 2. Exploded view of the gear: (a) high-speed rotor with $p_{h}=2$; (b) lowspeed rotor with $p_{l}=7$; (c) ferromagnetic pole-pieces with $Q=9$.

\section{MAGNETIC GEAR STRUCTURE AND ASSUMPTIONS}

\section{A. Structure of the Magnetic Gear}

The magnetic gear structure is shown in Fig. 1 and an exploded view is given in Fig. 2. As shown in Fig. 2, an axial magnetic gear consists of a high speed rotor with $p_{h}$ pole-pairs $\left(p_{h}=2\right)$, a low speed rotor with $p_{l}$ pole-pairs $\left(p_{l}=7\right)$, and $Q$ ferromagnetic pole-pieces placed between the two rotors $(Q=$ 9 ). The $Q$ ferromagnetic pole-pieces modulate the magnetic field created by the $p_{h}$ pole-pairs rotor (prime mover). The resultant magnetic field interacts with the $p_{l}$ pole-pairs rotor to transmit the torque to the load. From [1], the gear ratio $G_{r}$ between the low speed rotor $\Omega_{l}$ and the high speed rotors $\Omega_{h}$ (the ferromagnetic pole-pieces are kept stationary) is given by

$$
\Omega_{h}=G_{r} \Omega_{l} \quad \text { with } \quad G_{r}=\frac{p_{h}-Q}{p_{h}}=-\frac{p_{l}}{p_{h}}=-\frac{7}{2}
$$

The negative polarity for the gear ratio indicates that the low and high speed rotors contra-rotate.

\section{B. Modeling Assumptions}

Because of the three-dimensional nature of the magnetic field distribution in axial magnetic gear, rigorous computation of the torque requires a full three-dimensional analysis. However, the 3D analytical determination of the magnetic field in such structure is very difficult due to the presence of the pole-pieces as shown in Fig. 1. In order to simplify the analysis, the $3 \mathrm{D}$ problem of Fig. 1 is reduced to a $2 \mathrm{D}$ problem by unrolling the axial magnetic gear at the mean radius of the magnets $R_{m}=\left(R_{1}+R_{2}\right) / 2$. Fig. 3 shows the resulting $2 \mathrm{D}$ model which makes the axial magnetic gear equivalent to a linear magnetic gear with an infinite length in the perpendicular direction. The hypothesis adopted here suggests the use of the 2D Cartesian coordinates $(y, z)$ in which $y=R_{m} \theta$ as indicated in Fig. 3. In order to facilitate the analytical modeling, we adopt the following assumptions:

(1) The ferromagnetic parts (back iron and pole-pieces) are considered as perfect (i.e. infinite permeability), so they are considered as boundary condition for the studied problem (homogeneous Neumann boundary condition).

(2) The permanent magnets are axially magnetized with relative recoil permeability $\mu_{r}=1$.

Since the soft-magnetic material is considered as perfect (the field distribution is not calculated inside the ferromagnetic parts), we have to considered five regions as shown in Fig. 3: the PMs regions (I and IV), the air-gap regions (II and III) and the $Q$ slots regions (regions $i$ with $1<i<Q$ ).

A magnetic vector potential formulation is used here to describe the problem. The governing partial differential equations in each region are given by

$$
\left\{\begin{array}{l}
\nabla^{2} \boldsymbol{A}=-\mu_{0} \nabla \times \boldsymbol{M} \text { in Regions I and IV (PMs) } \\
\nabla^{2} \boldsymbol{A}=0 \quad \text { in Region II, III and } i \text { (air-gap and slots) }
\end{array}\right.
$$

with

$$
\boldsymbol{M}=M_{z} \boldsymbol{e}_{z}= \pm \frac{B_{r}}{\mu_{0}} \boldsymbol{e}_{z}
$$

where $\boldsymbol{M}$ is the magnetization vector and $B_{r}$ the remanence of the magnets. 
As shown in Fig. 3, the geometrical parameters of the magnetic gear are:

- for the high speed rotor: the magnets thickness $z_{1}$, the air gap length $\left(z_{2}-z_{1}\right)$, the pole-arc to pole-pitch ratio of the PMs $\alpha_{h}$;

- for the low speed rotor: the magnets thickness $\left(z_{5}-z_{4}\right)$, the air gap length $\left(z_{4}-z_{3}\right)$, the pole-arc to pole-pitch ratio of the PMs $\alpha_{l}$;

- for the ferromagnetic pole-pieces: the slots length $\left(z_{3}-z_{2}\right)$ and the slots opening angle $\beta$.

The angular position of the $i$-th slot is defined as

$$
\theta_{i}=-\frac{\beta}{2}+\frac{2 i \pi}{Q}+\theta_{0} \quad \text { with } \quad 1 \leq i \leq Q
$$

where $\theta_{0}$ is the initial angular position of the ferromagnetic pole-pieces.

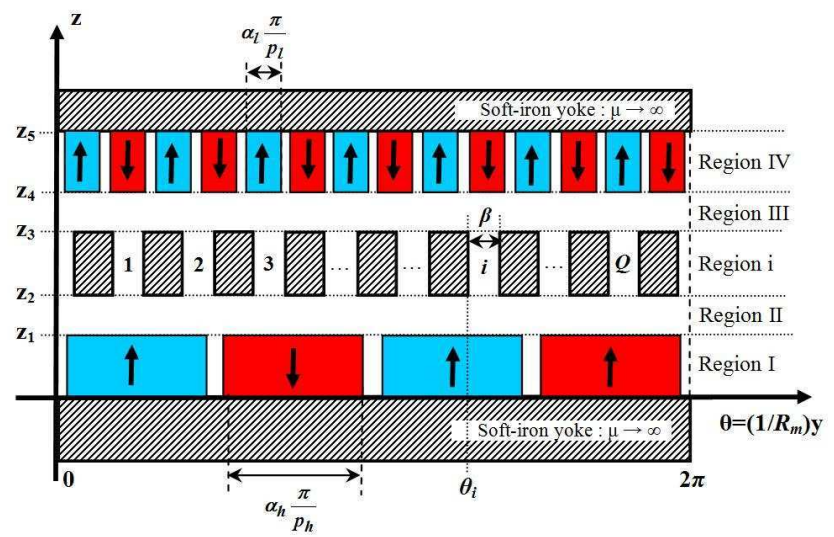

Fig. 3. 2-D model of the axial-type magnetic gear at the mean radius $R_{m}=\left(R_{1}+R_{2}\right) / 2$ for $p_{h}=2, p_{l}=7$ and $Q=9$.

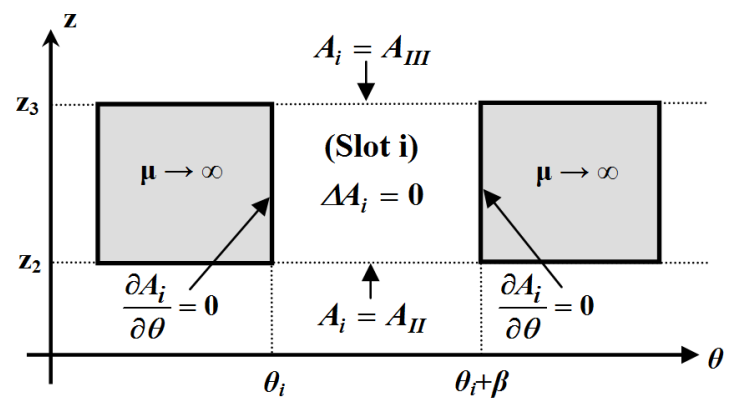

Fig. 4. $i$-th slot sub-domain with its boundary conditions.

\section{MAGNETIC FIELD CALCULATION}

We now develop the exact solution of the magnetic field in each region. As explained before, we focus more particularly on the solution of the magnetic field inside the slots as shown in Fig. 4. This is the main difficulty to solve. The magnetic field solutions in the air-gap and the magnet regions have been developed in [23] for the modeling of an axial magnetic coupling. From [23], only few changes on the boundary conditions and periodicity will be necessary here to obtain the solution of the magnetic vector potential in these regions.

\section{A. Magnetic Vector Potential in the i-th Slot Region}

The $i$-th slot region with its boundary conditions is shown in Fig. 4. In this region, we have to solve the Laplace equation (no source term in the slot). Using the Cartesian coordinates $(y, z)$ and replacing $y$ by $R_{m} \theta$, one obtains

$$
\frac{1}{R_{m}^{2}} \frac{\partial^{2} A_{i}}{\partial \theta^{2}}+\frac{\partial^{2} A_{i}}{\partial z^{2}}=0 \text { for }\left\{\begin{array}{l}
z_{2} \leq z \leq z_{3} \\
\theta_{i} \leq \theta \leq \theta_{i}+\beta
\end{array}\right.
$$

The mean radius $R_{m}$ has been explicitly put in (5) so the solutions will show its influence on the gear performances. We have supposed infinite permeability for the ferromagnetic pole-pieces, therefore the tangential component of the magnetic field at the sides of the slot is null. In terms of magnetic vector potential, we obtain homogeneous Neumann boundary conditions

$$
\left.\frac{\partial A_{i}}{\partial \theta}\right|_{\theta=\theta_{i}}=0 \quad \text { and }\left.\quad \frac{\partial A_{i}}{\partial \theta}\right|_{\theta=\theta_{i}+\beta}=0
$$

The continuity of the magnetic vector potential between the $i$-th slot and the air-gap regions I and II leads to

$$
A_{i}\left(\theta, z_{2}\right)=A_{I I}\left(\theta, z_{2}\right) \quad \text { and } \quad A_{i}\left(\theta, z_{3}\right)=A_{I I I}\left(\theta, z_{3}\right)
$$

where $A_{I I}(\theta, z)$ and $A_{I I I}(\theta, z)$ are the magnetic vector potentials in the air-gap regions.

In the method of separating variables, we write the solution of (5) under the form

$$
A_{i}(\theta, z)=\Theta_{i}(\theta) Z_{i}(z)
$$

which is a product of two functions, each depending only on one of the variables $\theta$ and $z$. By inserting (8) into (5) and introducing the classical separation constant $\lambda$, we obtain two ordinary linear differential equations

$$
\begin{gathered}
\Theta_{i}^{\prime \prime}+\lambda \Theta_{i}=0 \\
Z_{i}^{\prime \prime}-\left(\lambda / R_{m}^{2}\right) Z_{i}=0
\end{gathered}
$$

From the boundary conditions (6), it follows that

$$
\Theta_{i}^{\prime}\left(\theta_{i}\right)=0 \text { and } \Theta_{i}^{\prime}\left(\theta_{i}+\beta\right)=0
$$

We have to find the values of the parameter $\lambda$ for which non-trivial solutions of (9) satisfying the boundary conditions (11) exist [26]. The eigenvalues of the problem (9), (11) are 


$$
\begin{aligned}
& \lambda_{0}=0 \\
& \lambda_{k}=\left(\frac{k \pi}{\beta}\right)^{2} \text { with } k=1,2,3, \ldots . .
\end{aligned}
$$

Note that, in this problem, the eigenvalue zero exists because of the Neumann boundary conditions (6).

The eigenfunctions corresponding to $\lambda_{0}$ and $\lambda_{k}$ are given by

$$
\begin{aligned}
& \Theta_{i 0}(\theta)=1 \\
& \Theta_{i k}(\theta)=\cos \left(\frac{k \pi}{\beta}\left(\theta-\theta_{i}\right)\right)
\end{aligned}
$$

For $\lambda_{0}$ and $\lambda_{k}$, the general solutions of (10) are

$$
\begin{gathered}
Z_{i 0}(z)=a_{0}^{i}+b_{0}^{i} z \\
Z_{i k}(z)=A_{k}^{i} e^{-\frac{k \pi}{\beta R_{m}} z}+B_{k}^{i} e^{\frac{k \pi}{\beta R_{m}} z}
\end{gathered}
$$

where $a_{0}^{i}, b_{0}^{i}, A_{k}^{i}$, and $B_{k}^{i}$ are integration constants. The general solution of (5) is written in terms of infinite series

$$
\begin{aligned}
& A_{i}(\theta, z)=a_{0}^{i}+b_{0}^{i} z \\
& +\sum_{k=1}^{\infty}\left(A_{k}^{i} e^{-\frac{k \pi}{\beta R_{m}} z}+B_{k}^{i} e^{\frac{k \pi}{\beta R_{m}} z}\right) \cos \left(\frac{k \pi}{\beta}\left(\theta-\theta_{i}\right)\right)
\end{aligned}
$$

Considering the interface conditions (7), the general solution of the magnetic vector potential in the $i$-th slot domain can be rewritten as

$$
\begin{aligned}
& A_{i}(\theta, z)=a_{0}^{i}+b_{0}^{i} z \\
& \sum_{k=1}^{\infty}\left(a_{k}^{i} \frac{\operatorname{sh}\left(\frac{k \pi}{\beta R_{m}}\left(z-z_{3}\right)\right)}{\operatorname{sh}\left(\frac{k \pi}{\beta R_{m}}\left(z_{2}-z_{3}\right)\right)}+b_{k}^{i} \frac{\operatorname{sh}\left(\frac{k \pi}{\beta R_{m}}\left(z-z_{2}\right)\right)}{\operatorname{sh}\left(\frac{k \pi}{\beta R_{m}}\left(z_{3}-z_{2}\right)\right)}\right) \cos \left(\frac{k \pi}{\beta}\left(\theta-\theta_{i}\right)\right)
\end{aligned}
$$

where $a_{k}^{i}$ and $b_{k}^{i}$ are new integration constants. The integration constants can be determined using a Fourier series expansion of air-gap magnetic vector potentials $A_{I I}\left(\theta, z_{2}\right)$ and $A_{I I I}\left(\theta, z_{3}\right)$ over the $i^{\text {th }}$ slot interval $\left[\theta_{i}, \theta_{i}+\beta\right]$.

$$
\begin{aligned}
& a_{0}^{i}+b_{0}^{i} z_{2}=\frac{1}{\beta} \int_{\theta_{i}}^{\theta_{i}+\beta} A_{I I}\left(\theta, z_{2}\right) d \theta \\
& a_{0}^{i}+b_{0}^{i} z_{3}=\frac{1}{\beta} \int_{\theta_{i}}^{\theta_{i}+\beta} A_{I I I}\left(\theta, z_{3}\right) d \theta
\end{aligned}
$$

$$
\begin{aligned}
& a_{k}^{i}=\frac{2}{\beta} \int_{\theta_{i}}^{\theta_{i}+\beta} A_{I I}\left(\theta, z_{2}\right) \cos \left(\frac{k \pi}{\beta}\left(\theta-\theta_{i}\right)\right) d \theta \\
& b_{k}^{i}=\frac{2}{\beta} \int_{\theta_{i}}^{\theta_{i}+\beta} A_{I I I}\left(\theta, z_{3}\right) \cos \left(\frac{k \pi}{\beta}\left(\theta-\theta_{i}\right)\right) d \theta
\end{aligned}
$$

The expressions of constants $a_{0}^{i}, b_{0}^{i}, a_{k}^{i}$ and $b_{k}^{i}$ are developed in the appendix.

The term $b_{0}^{i} z$ which appears in the general solutions (19) is of great importance. Indeed, neglecting this term can result in a wrong evaluation of the other Fourier coefficients (see the appendix) and therefore in the prediction of the magnetic gear performances. This term corresponds to a nonzero mean value for the tangential component of the flux density $\left(\partial A_{i} / \partial z\right)$, even without source term in the slots (as shown in Fig. 7b). This result is different from the one given in [22] where it is indicated that the tangential component of the flux density in a slot open on two sides has an extra constant unknown (called $B_{0}$ in [22]) only if a source term with a dc component is present in the slot (current density or tangential magnetization). Moreover, compared to [22], we have a supplementary integration constant $\left(a_{0}^{i}\right)$ to determine. This supplementary constant has no impact on the flux density and therefore on the torque value (we have to derivate the magnetic vector potential to compute the flux density and this constant disappears). However, the constants $\left(a_{0}^{i}\right)$ (in each slot) can be used to compute the back-EMF if coils are placed around the ferromagnetic pole-pieces to extract output electrical power as it has been proposed recently [24].

\section{B. Magnetic Vector Potential in the Magnet Regions}

In the magnets region (region I), we have to solve the following equation

$$
\frac{1}{R_{m}^{2}} \frac{\partial^{2} A_{I}}{\partial \theta^{2}}+\frac{\partial^{2} A_{I}}{\partial z^{2}}=-\frac{\mu_{0}}{R_{m}} \frac{\partial M_{z}}{\partial \theta} \quad \text { for }\left\{\begin{array}{l}
0 \leq z \leq z_{1} \\
0 \leq \theta \leq 2 \pi
\end{array}\right.
$$

where $\mu_{0}$ is the permeability of the vacuum and $M_{z}$ is the axial magnetization of the magnets.

The distribution of axial magnetization $M_{z}$ can be expressed in Fourier's series [23] and replaced in (24)

$$
\begin{gathered}
M_{z}(\theta)=\sum_{n=1}^{\infty} M_{n} \sin \left((2 n-1) p_{h}\left(\theta-\delta_{h}\right)\right) \\
M_{n}=\frac{4 B_{r}}{(2 n-1) \pi \mu_{0}} \cos \left((2 n-1) \frac{\pi}{2}\left(1-\alpha_{h}\right)\right) \text { with } n=1,2,3,4 \ldots
\end{gathered}
$$

where $\delta_{h}$ is the initial phase angle of the high speed rotor.

The general solution for the magnetic vector potential in region I can be obtained by using the same procedure as the one developed in [23]. However, in [23] the solution was given for a magnetic coupling where the solution presents a spatial periodicity in the $\theta$-direction $(2 \pi / \mathrm{p})$ which is not the case for the magnetic gear studied here. Therefore, the solution in the magnet region (region I of Fig. 3) is given by 


$$
\begin{aligned}
A_{I}(\theta, z) & =\sum_{n=1}^{\infty}\left(a_{n}^{I} \frac{\operatorname{ch}\left(\frac{n}{R_{m}} z\right)}{\operatorname{ch}\left(\frac{n}{R_{m}} z_{1}\right)}+K_{n}^{h} \cos \left(n \delta_{h}\right)\right) \cos (n \theta) \\
& +\sum_{n=1}^{\infty}\left(\frac{\operatorname{ch}\left(\frac{n}{R_{m}} z\right)}{c_{n}^{I} \frac{n}{\operatorname{ch}\left(\frac{n}{R_{m}} z_{1}\right)}+K_{n}^{h} \sin \left(n \delta_{h}\right)}\right) \sin (n \theta)
\end{aligned}
$$

with

$$
K_{n}^{h}=\left\{\begin{array}{l}
\frac{4 B_{r} R_{m} p_{h}}{\pi n^{2}} \cos \left(n \frac{\pi}{2 p_{h}}\left(1-\alpha_{h}\right)\right) \text { for } n=j p_{h} ; j=1,3,5, \ldots \\
0 \quad \text { otherwise }
\end{array}\right.
$$

The integration constants $a_{n}^{I}$ and $c_{n}^{I}$ are determined using a Fourier series expansion of $A_{I I}\left(\theta, z_{1}\right)$ over the interval $[0,2 \pi]$

$$
\begin{aligned}
& a_{n}^{I}+K_{n}^{h} \cos \left(n \delta_{h}\right)=\frac{2}{2 \pi} \int_{0}^{2 \pi} A_{I I}\left(\theta, z_{1}\right) \cos (n \theta) d \theta \\
& c_{n}^{I}+K_{n}^{h} \sin \left(n \delta_{h}\right)=\frac{2}{2 \pi} \int_{0}^{2 \pi} A_{I I}\left(\theta, z_{1}\right) \sin (n \theta) d \theta
\end{aligned}
$$

The expressions of constants $a_{n}^{I}$ and $c_{n}^{I}$ are given in the appendix.

The same method is used to find the solution in region IV (low speed rotor PMs). This leads to the following expression for the magnetic vector potential

$$
\begin{aligned}
A_{I V}(\theta, z) & =\sum_{n=1}^{\infty}\left(a_{n}^{I V} \frac{\operatorname{ch}\left(\frac{n}{R_{m}}\left(z-z_{5}\right)\right)}{\operatorname{ch}\left(\frac{n}{R_{m}}\left(z_{4}-z_{5}\right)\right)}+K_{n}^{l} \cos \left(n \delta_{l}\right)\right) \cos (n \theta) \\
& +\sum_{n=1}^{\infty}\left(c_{n}^{I V} \frac{\operatorname{ch}\left(\frac{n}{R_{m}}\left(z-z_{5}\right)\right)}{c h\left(\frac{n}{R_{m}}\left(z_{4}-z_{5}\right)\right)}+K_{n}^{l} \sin \left(n \delta_{l}\right)\right) \sin (n \theta)
\end{aligned}
$$

with

$$
K_{n}^{l}=\left\{\begin{array}{l}
\frac{4 B_{r} R_{m} p_{l}}{\pi n^{2}} \cos \left(n \frac{\pi}{2 p_{l}}\left(1-\alpha_{l}\right)\right) \text { for } n=j p_{l} ; j=1,3,5, \ldots \\
0 \quad \text { otherwise }
\end{array}\right.
$$

Integration constants $a_{n}^{I V}$ and $c_{n}^{I V}$ in (31) are determined using a Fourier series expansion of $A_{I I I}\left(\theta, z_{4}\right)$ over the interval $[0,2 \pi]$

$$
a_{n}^{I V}+K_{n}^{l} \cos \left(n \delta_{l}\right)=\frac{2}{2 \pi} \int_{0}^{2 \pi} A_{I I I}\left(\theta, z_{4}\right) \cos (n \theta) d \theta
$$

$$
c_{n}^{I V}+K_{n}^{l} \sin \left(n \delta_{l}\right)=\frac{2}{2 \pi} \int_{0}^{2 \pi} A_{I I I}\left(\theta, z_{4}\right) \sin (n \theta) d \theta
$$

The expressions of constants $a_{n}^{I V}$ and $c_{n}^{I V}$ are given in the appendix.

\section{Magnetic Vector Potential in the Air-Gap Regions}

For the air-gap region (region II), we have to solve the Laplace equation

$$
\frac{1}{R_{m}^{2}} \frac{\partial^{2} A_{I I}}{\partial \theta^{2}}+\frac{\partial^{2} A_{I I}}{\partial z^{2}}=0 \text { for }\left\{\begin{array}{l}
z_{1} \leq z \leq z_{2} \\
0 \leq \theta \leq 2 \pi
\end{array}\right.
$$

To find the general solution for the magnetic vector potential in the air-gap region, we follow the same method as the one developed in [23]. However, as for the magnet regions, the solution presents no spatial periodicity in the $\theta$-direction and in addition, the interface condition at $z=z_{2}$ is more difficult because of the existence of the slots as shown in Fig. 3.

Therefore, at $z=z_{2}$, we have to consider the following interface condition (continuity of the tangential magnetic field at the interface between the ferromagnetic pole-pieces and the air-gap and zero value for the tangential magnetic field elsewhere i.e. infinite permeability of the ferromagnetic polepieces)

$$
\left.\frac{\partial A_{I I}}{\partial z}\right|_{z=z_{2}}= \begin{cases}\left.\frac{\partial A_{i}}{\partial z}\right|_{z=z_{2}} & \forall \theta \in\left[\theta_{i}, \theta_{i}+\beta\right] \\ 0 & \text { elsewhere }\end{cases}
$$

where $A_{i}(r, \theta)$ is the magnetic vector potential in the $i$-th slot given by (19).

The continuity of the tangential component of the magnetic field at $z=z_{1}$ gives

$$
\left.\frac{\partial A_{I I}}{\partial z}\right|_{z=z_{1}}=\left.\frac{\partial A_{I}}{\partial z}\right|_{z=z_{1}}
$$

After some calculus, the general solution of the magnetic vector potential in the air-gap can be written as

$$
\begin{aligned}
& A_{I I}(\theta, z)= \\
& \sum_{n=1}^{\infty}\left(a_{n}^{I I} \frac{R_{m}}{n} \frac{\operatorname{ch}\left(\frac{n}{R_{m}}\left(z-z_{2}\right)\right)}{\operatorname{sh}\left(\frac{n}{R_{m}}\left(z_{1}-z_{2}\right)\right)}+b_{n}^{I I} \frac{R_{m}}{n} \frac{\operatorname{ch}\left(\frac{n}{R_{m}}\left(z-z_{1}\right)\right)}{\operatorname{sh}\left(\frac{n}{R_{m}}\left(z_{2}-z_{1}\right)\right)}\right) \cos (n \theta) \\
& +\sum_{n=1}^{\infty}\left(c_{n}^{I I} \frac{R_{m}}{n} \frac{\operatorname{ch}\left(\frac{n}{R_{m}}\left(z-z_{2}\right)\right)}{\operatorname{sh}\left(\frac{n}{R_{m}}\left(z_{1}-z_{2}\right)\right)}+d_{n}^{I I} \frac{R_{m}}{n} \frac{\operatorname{ch}\left(\frac{n}{R_{m}}\left(z-z_{1}\right)\right)}{\operatorname{sh}\left(\frac{n}{R_{m}}\left(z_{2}-z_{1}\right)\right)}\right) \sin (n \theta)
\end{aligned}
$$


The integration constants $a_{n}^{I I}, \quad b_{n}^{I I}, \quad c_{n}^{I I}$ and $d_{n}^{I I}$ are determined as follows (Fourier series expansion)

$$
\begin{aligned}
& a_{n}^{I I}=\left.\frac{2}{2 \pi} \int_{0}^{2 \pi} \frac{\partial A_{I}}{\partial z}\right|_{z_{1}} \cos (n \theta) d \theta \\
& b_{n}^{I I}=\left.\frac{2}{2 \pi} \sum_{i=1}^{Q} \int_{\theta_{i}}^{\theta_{i}+\beta} \frac{\partial A_{i}}{\partial z}\right|_{z=z_{2}} \cos (n \theta) d \theta \\
& c_{n}^{I I}=\left.\frac{2}{2 \pi} \int_{0}^{2 \pi} \frac{\partial A_{I}}{\partial z}\right|_{z_{1}} \sin (n \theta) d \theta \\
& d_{n}^{I I}=\left.\frac{2}{2 \pi} \sum_{i=1}^{Q} \int_{\theta_{i}}^{\theta_{i}+\beta} \frac{\partial A_{i}}{\partial z}\right|_{z=z_{2}} \sin (n \theta) d \theta
\end{aligned}
$$

The expressions of these constants are developed in the appendix.

As explained in [26], the Neumann problem (35), (36) and (37) is well defined only if

$$
\left.\int_{0}^{2 \pi} \frac{\partial A_{I}}{\partial z}\right|_{z_{1}} d \theta=\int_{0}^{2 \pi} f(\theta) d \theta
$$

The treatment of (43) gives an additional constraint between the coefficients $b_{0}^{i}$ defined in (19)

$$
\sum_{i=1}^{Q} b_{0}^{i}=0
$$

The axial and tangential components of the magnetic flux density in air-gap region II can be deduced from magnetic vector potential (38) by

$$
B_{I I z}=-\frac{1}{R_{m}} \frac{\partial A_{I I}}{\partial \theta} \quad B_{I I \theta}=\frac{\partial A_{I I}}{\partial z}
$$

The same procedure is followed to obtain the solution in region III (air-gap near low speed rotor). This leads to the following expression for the magnetic vector potential

$$
\begin{aligned}
& A_{I I I}(\theta, z)= \\
& \sum_{n=1}^{\infty}\left(a_{n}^{I I I} \frac{R_{m}}{n} \frac{\operatorname{ch}\left(\frac{n}{R_{m}}\left(z-z_{4}\right)\right)}{\operatorname{sh}\left(\frac{n}{R_{m}}\left(z_{3}-z_{4}\right)\right)}+b_{n}^{I I I} \frac{R_{m}}{n} \frac{\operatorname{ch}\left(\frac{n}{R_{m}}\left(z-z_{3}\right)\right)}{\operatorname{sh}\left(\frac{n}{R_{m}}\left(z_{4}-z_{3}\right)\right)}\right) \cos (n \theta) \\
& +\sum_{n=1}^{\infty}\left(c_{n}^{I I I} \frac{R_{m}}{n} \frac{\operatorname{ch}\left(\frac{n}{R_{m}}\left(z-z_{4}\right)\right)}{\operatorname{sh}\left(\frac{n}{R_{m}}\left(z_{3}-z_{4}\right)\right)}+d_{n}^{I I I} \frac{R_{m}}{n} \frac{\operatorname{ch}\left(\frac{n}{R_{m}}\left(z-z_{3}\right)\right)}{\operatorname{sh}\left(\frac{n}{R_{m}}\left(z_{4}-z_{3}\right)\right)}\right) \sin (n \theta)
\end{aligned}
$$

with

$$
\begin{aligned}
& a_{n}^{I I I}=\left.\frac{2}{2 \pi} \sum_{i=1}^{Q} \int_{\theta_{i}}^{\theta_{i}+\beta} \frac{\partial A_{i}}{\partial z}\right|_{z=z_{3}} \cos (n \theta) d \theta \\
& b_{n}^{I I I}=\left.\frac{2}{2 \pi} \int_{0}^{2 \pi} \frac{\partial A_{I V}}{\partial z}\right|_{z_{4}} \cos (n \theta) d \theta \\
& c_{n}^{I I I}=\left.\frac{2}{2 \pi} \sum_{i=1}^{Q} \int_{\theta_{i}}^{\theta_{i}+\beta} \frac{\partial A_{i}}{\partial z}\right|_{z=z_{3}} \sin (n \theta) d \theta \\
& d_{n}^{I I I}=\left.\frac{2}{2 \pi} \int_{0}^{2 \pi} \frac{\partial A_{I V}}{\partial z}\right|_{z_{4}} \sin (n \theta) d \theta
\end{aligned}
$$

The expressions of the constants $a_{n}^{I I I}, b_{n}^{I I I}, c_{n}^{I I I}$ and $d_{n}^{I I I}$ are given in the appendix.

\section{Electromagnetic Torque and Axial Force Expressions}

The electromagnetic torque and the axial force applied on the high speed rotor are obtained using the classical Maxwell stress tensor. The integration line can arbitrarily be any closed line in the air-gap region i.e. $z=\zeta \in\left[z_{1}, z_{2}\right]$. By considering the $2 \mathrm{D}$ approximation of the magnetic field distribution, we obtain the following relations for the torque and the axial force.

$$
\begin{aligned}
& T_{h}=\frac{R_{2}^{3}-R_{1}^{3}}{3 \mu_{0}} \int_{0}^{2 \pi} B_{I I \theta}(\theta, \zeta) B_{I I z}(\theta, \zeta) d \theta \\
& F_{h}=\frac{R_{2}^{2}-R_{1}^{2}}{4 \mu_{0}} \int_{0}^{2 \pi}\left(B_{I I z}^{2}(\theta, \zeta)-B_{I I \theta}^{2}(\theta, \zeta)\right) d \theta
\end{aligned}
$$

Substituting (45) into (51) and (52), the analytical expression for the torque and the axial force can be obtained directly from the constant integrations

$$
\begin{aligned}
& T_{h}=\frac{\pi\left(R_{2}^{3}-R_{1}^{3}\right)}{3 \mu_{0}} \sum_{n=1}^{\infty}\left(W_{n} X_{n}+Y_{n} Z_{n}\right) \\
& F_{h}=\frac{\pi\left(R_{2}^{2}-R_{1}^{2}\right)}{4 \mu_{0}} \sum_{n=1}^{\infty}\left(\left(Z_{n}+X_{n}\right)^{2}-\left(W_{n}+Y_{n}\right)^{2}\right)
\end{aligned}
$$

where the coefficients $W_{n}, X_{n}, Y_{n}$ and $Z_{n}$ are given in the appendix.

Similar expressions can be obtained for the electromagnetic torque and the axial force applied on the low speed rotor. 


\section{ANALYTICAL RESULTS AND COMPARISON WITH FINITE ELEMENT SIMULATIONS}

In order to validate the 2D analytical model and show its limits, we compared the results obtained with the proposed model and those obtained with 3D finite element simulations (COMSOL ${ }^{\circledR}$ Multiphysics software). The numerical model has been performed with the same assumptions as the analytical model (iron parts with a relative permeability $\mu_{r}=1000$ and axially magnetized magnets with $\mu_{r}=1$ ). For the analytical model, we have limited the infinite series solutions by using a finite number of harmonic terms $N$ and $K$ as indicated in Table I. A number of 50 harmonic terms is sufficient to predict the magnetic field distribution with a good accuracy.

For the simulations, we have considered the geometrical parameters given in Table I. These parameters have not been optimized. The aim of this paper is to show the advantages and limitations of the proposed model. However, the influence of some geometrical parameters (i.e. magnets and pole-pieces thicknesses) on the magnetic gear performances has been investigated here to show the effectiveness of the proposed model.

\section{A. Flux Density Distribution}

Fig. 5 and Fig. 6 respectively show the flux density distributions (axial and tangential components) in the middle of the high-speed air-gap region $(z=8 \mathrm{~mm})$ and low-speed airgap region $(z=20 \mathrm{~mm})$. The phase angles $\delta_{h}$ and $\delta_{l}$ of the high-speed and low-speed rotors are fixed to zero. The initial position of the ferromagnetic pole-pieces is fixed to $\theta_{0}=-$ $\pi /(2 Q)$ that corresponds to the pull-out torque position.

From Fig. 5 and Fig. 6, the effects of the ferromagnetic pole-pieces on the magnetic field distribution in the air-gap regions are very clear. The magnetic field is modulated by the presence of the ferromagnetic pole-pieces. As the magnetic flux density is calculated at mean radius $R_{m}$, we can observe good agreements between 3D FEM results and those obtained with the $2 \mathrm{D}$ analytical model.

TABLE I

PARAMETERS OF THE STUDIED AXIAL FIELD MAGNETIC GEAR

\begin{tabular}{lll}
\hline \hline \multirow{2}{*}{ Symbol } & \multicolumn{1}{c}{ Quantity } & value \\
\hline$R_{1}$ & Inner radius of the magnets & $30 \mathrm{~mm}$ \\
$R_{2}$ & Outer radius of the magnets & $70 \mathrm{~mm}$ \\
$z_{1}$ & Magnets thickness (high-speed rotor) & $7 \mathrm{~mm}$ \\
$z_{2}-z_{1}$ & Air-gap length (near the high speed rotor) & $2 \mathrm{~mm}$ \\
$z_{3}-z_{2}$ & Length of the ferromagnetic pole-pieces & $10 \mathrm{~mm}$ \\
$z_{4}-z_{3}$ & Air-gap length (near the low-speed rotor) & $2 \mathrm{~mm}$ \\
$z_{5}-z_{4}$ & Magnet thickness (low-speed rotor) & $7 \mathrm{~mm}$ \\
$B_{r}$ & Remanence of the magnets & $1.25 \mathrm{~T}$ \\
$\alpha_{h}$ & Pole-arc to pole-pitch ratio of high-speed PMs & 1 \\
$\alpha_{l}$ & Pole-arc to pole-pitch ratio of low-speed PMs & 1 \\
$p_{h}$ & Pole-pairs number of high speed rotor & 2 \\
$p_{l}$ & Pole-pairs number of low speed rotor & 7 \\
$Q$ & Number of ferromagnetic pole-pieces & 9 \\
$\beta$ & Slot opening & $\pi / Q \mathrm{rad}$ \\
$K$ & Number of harmonics used for magnetic field & 50 \\
& calculation in the air-gap and PMs domains & \\
$N$ & Number of harmonics used for magnetic field & 50 \\
\hline \hline
\end{tabular}

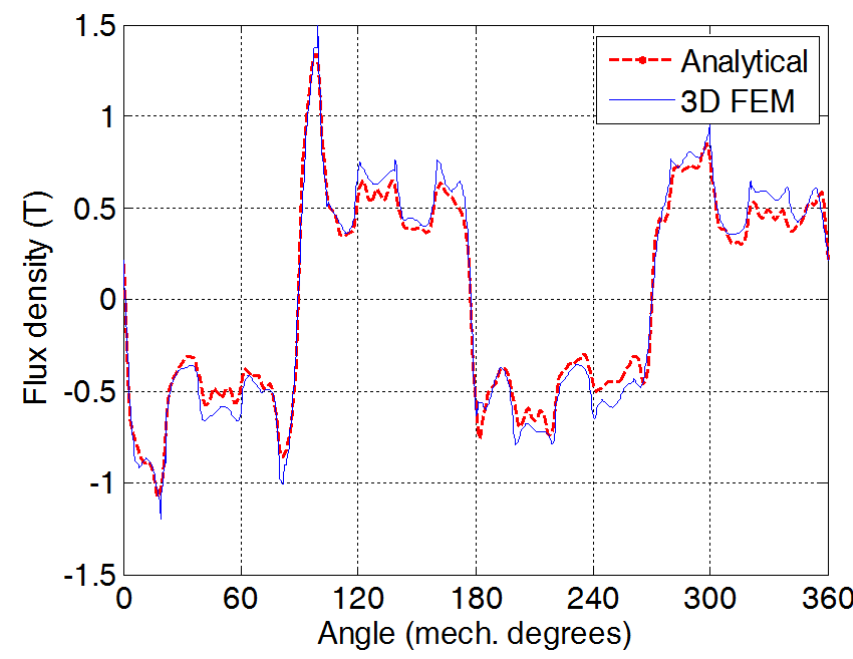

(a)

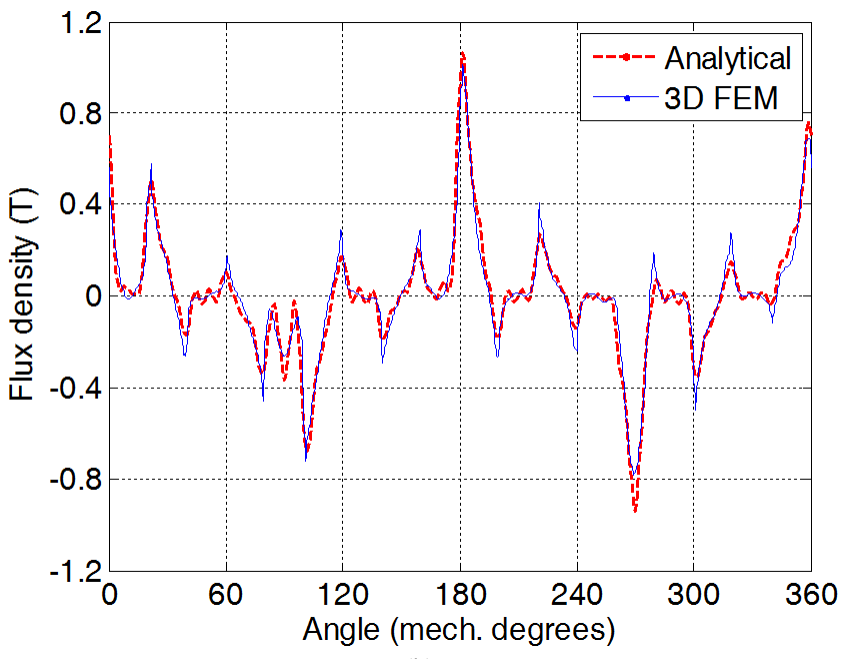

(b)

Fig. 5. Flux density distribution in the middle of the high-speed air-gap $(z=8$ $\mathrm{mm}$ ): (a) axial component (b) tangential component.

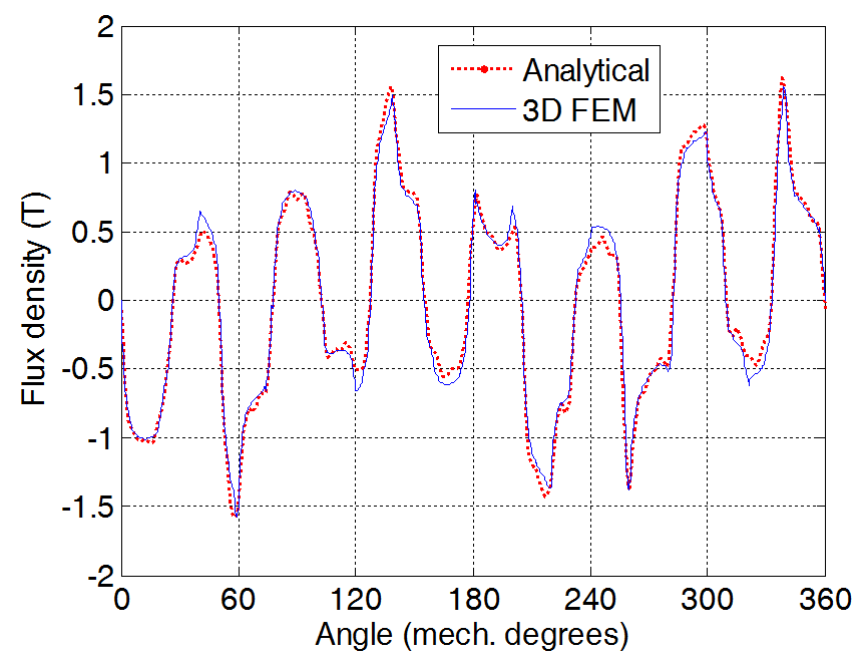

(a) 


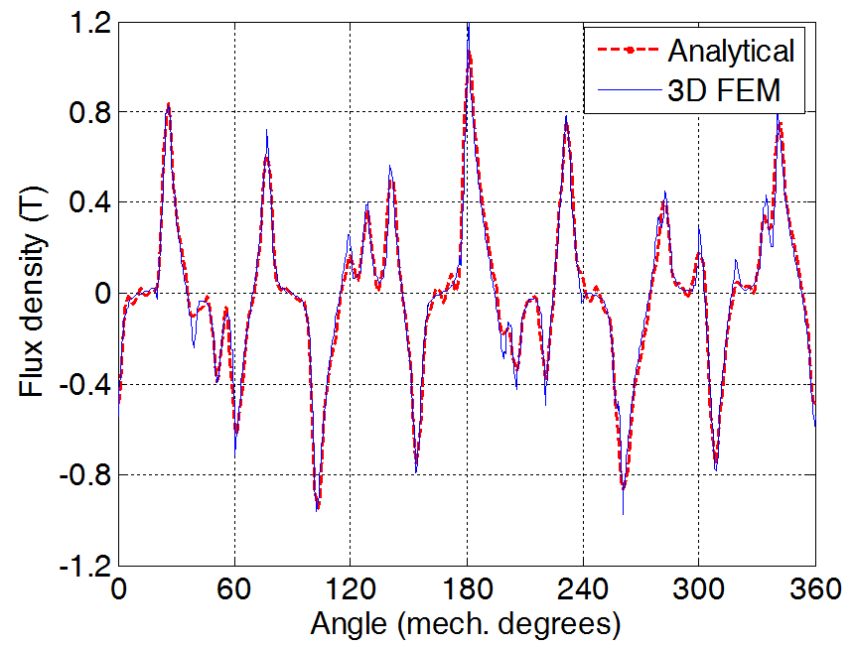

(b)

Fig. 6. Flux density distribution in the middle of the low-speed air-gap ( $z=$ $20 \mathrm{~mm}$ ): (a) radial component (b) tangential component.

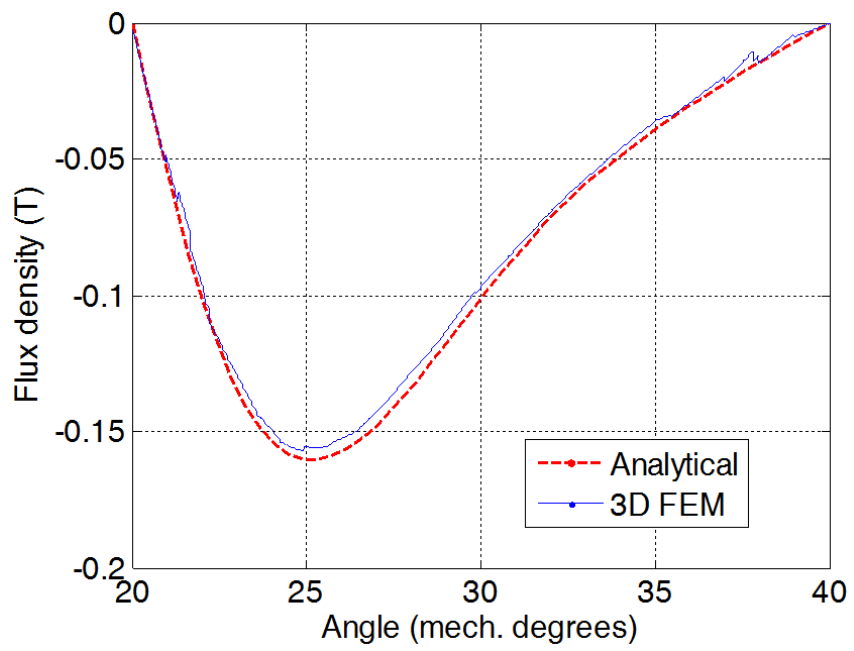

(a)

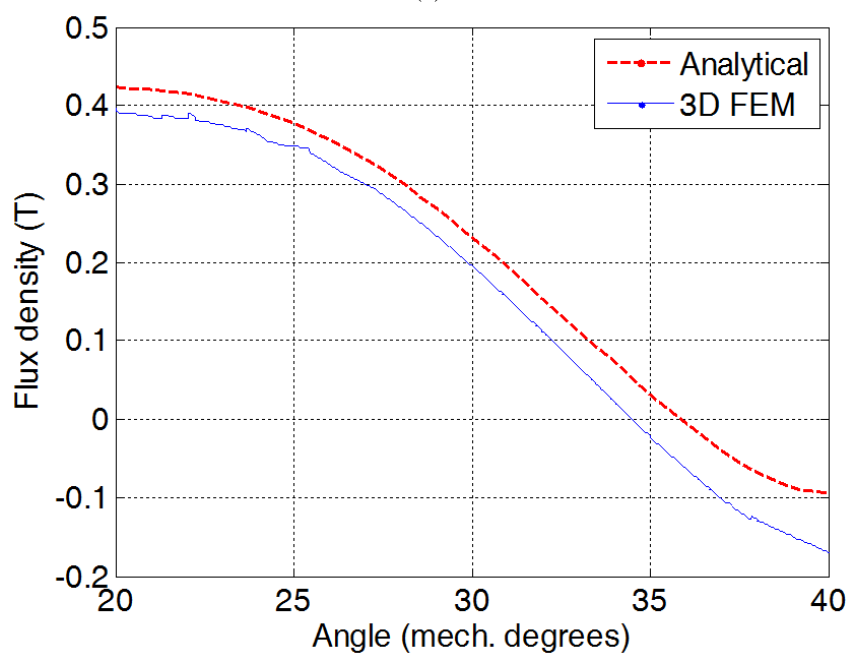

(b)

Fig. 7. Flux density distribution in the middle of the slot $i=1(z=14 \mathrm{~mm}$; $20^{\circ}<\theta<40^{\circ}$ ): (a) axial component (b) tangential component.
Using (19), the axial and tangential components of the flux density in the $1^{\text {st }}$ slots region $\left(i=1 ; z=14 \mathrm{~mm}\right.$ and $\left.20^{\circ}<\theta<40^{\circ}\right)$ are calculated and plotted in Fig. 7. In accordance with the boundary conditions (6) used in the analytical model, one can observe in Fig. 7a that the axial component of the flux density is equal to zero at the iron boundaries $\left(\theta=20^{\circ}\right.$ and $\left.40^{\circ}\right)$. It can be seen that the proposed analytical model can predict the flux density in the slot regions (at the mean radius) with a good precision. The difference between $2 \mathrm{D}$ analytical predictions and 3D FE simulations is less than $5 \%$ for the axial component and is about $15 \%$ for the tangential component. Moreover, as discussed in the introduction and in sub-section III.A, the tangential component of the flux density presents a mean value of around $0.2 \mathrm{~T}$ which corresponds to a nonzero value for the coefficient $b_{0}^{i}$ given in (19).

\section{B. Torque and Axial Force}

Fig. 8a shows the static torque on the high-speed rotor with the ferromagnetic pole-pieces and the low-speed rotor fixed $\left(\theta_{0}=\delta_{l}=0^{\circ}\right)$. The phase angle of the high-speed rotor $\delta_{h}$ varies from $0^{\circ}$ to $90^{\circ}$ (i.e. $180^{\circ} / p_{h}$ ). The same operation is done for the low-speed rotor with $\delta_{l}$ varying from $0^{\circ}$ to $25.7^{\circ}$ (i.e. $180^{\circ} / p_{l}$ ) and the result is given in Fig. 8b. As expected for this type of device [23], the 2D analytical model gives higher torque values when compared to the 3D FE simulations. It can be seen in Fig. 8 that the error on the pull-out torque prediction is about $30 \%$. For the high-speed rotor, the torque value obtained with the analytical model is about $13 \mathrm{Nm}$ whereas the 3D FE simulations give $10 \mathrm{Nm}$. For the low-speed rotor, the torque values are about $46 \mathrm{Nm}$ (analytical model) and $35 \mathrm{Nm}$ (3D FEM). These differences are due to the 3-D effects which are neglected in the proposed analytical model. We can observe in Fig. 8a important torque ripples on the high speed rotor. This is due to low order harmonics in the flux density distribution in the high speed air-gap. This effect can be reduced by properly choosing $p_{h}$ and $Q$ for a given gear ratio [1]. We can observe that the gear ratio is the same for analytical and FE results $\left(G_{r}=46 / 13 \approx 35 / 10=3.5\right)$ and, as expected, is equal to $p_{l} / p_{h}(1)$.

To show the influence of the number of harmonic terms used in the analytical computation, Fig. 9a and Fig. 9b respectively give the maximum torque (low speed rotor) and the computation time vs. the number of harmonic terms. We consider the same number of harmonic terms for all subdomains $(\mathrm{N}=\mathrm{K})$. For the studied example, one can observe that a number of 30 harmonic terms is sufficient to predict the torque with a good precision. With this choice, Fig. $9 \mathrm{~b}$ shows that the analytical computation takes about $0.15 \mathrm{~s}$ whereas the linear 3D FEM simulation, with a magnetic scalar formulation, lasts 40s. The analytical computations being much faster, the presented model can advantageously be used in a preliminary design of axial magnetic gears.

Fig. 10 shows the variation of the axial force acting on the high-speed and low-speed rotors as a function of respectively angular displacements $\delta_{h}$ and $\delta_{l}$. As shown in Fig. 10, the two 
rotors are attracted by the ferromagnetic pole pieces (we have a positive axial force for the high speed rotor and a negative axial force for the low speed rotor). The maximal force on both rotors are obtained for $\delta_{h}=\delta_{l}=\theta_{0}=0^{\circ}$. The error between analytical predictions and FE simulations on the maximal axial force is less important for the high speed rotor (less than 5\%) than for the low speed rotor (about 15\%).

\section{Influence of Magnet and Pole-Pieces Thicknesses}

The magnets thicknesses (high-speed and low-speed rotors) as well as the ferromagnetic pole-pieces thickness have a significant influence on the performances of axial magnetic gears.

We first study the effect of the pole-pieces thickness $\left(z_{3}-z_{2}\right)$ on the torque value (low-speed rotor). The other geometrical parameters don't change and are the ones indicated in Table I. The pole-pieces thickness varies from $4 \mathrm{~mm}$ to $20 \mathrm{~mm}$. Fig. 11 shows the torque as a function of the pole-pieces thickness. The torque has been computed for $\delta_{l}=0.75 * 90 \% p_{l}$ (i.e. $9.64^{\circ}$ ). At this position, one can observe from Fig. 11 that the maximal torque value is obtained for a pole-piece thickness of $12 \mathrm{~mm}$. As mentioned previously, Fig. 11 also shows that the analytical model predicts torque values $30 \%$ higher than the 3D-FEM computations. However, it is important to note that the analytical and the numerical computations follow the same trend and they give the same result for the optimum value of the pole-pieces thickness.

We now study the effect of the magnets thicknesses (low speed and high speed rotors) on the torque. We fix the total thickness of the magnets $z_{1}+\left(z_{5}-z_{4}\right)=14 \mathrm{~mm}$. We define the ratio between the magnets thickness of the high speed rotor and the total thickness of the magnets as

$$
K_{m}=\frac{z_{1}}{z_{1}+\left(z_{5}-z_{4}\right)}
$$

For the parametric study, $K_{m}$ varies from 0.2 to 0.8 and the other geometrical parameters are the ones given in Table I. Fig. 12 shows the torque as a function of $K_{m}$ obtained with the analytical model and with 3D finite element simulations. Once again, we can observe that the analytical and the numerical computations follow the same trend and give the same result for the optimum value of $K_{m}\left(K_{m}=0.6\right)$. For the studied example, this result shows that, the thickness of the high-speed magnets must be higher $(8.4 \mathrm{~mm})$ than the thickness of the low-speed magnets $(5.6 \mathrm{~mm})$. This parametric study has not been investigated further, nevertheless, for a given total magnets volume, the torque transmission capabilities of an axial field magnetic gear are better when the high-speed rotor magnet volume is higher than the low-speed rotor magnet volume (ie. $\mathrm{K}_{\mathrm{m}}>0.5$ ).

We have shown through these two examples that the analytical model is suitable in the determination of optimum geometrical parameters values that maximise the torque. This is of great benefit in any optimisation study since the analytical computation is very quick.

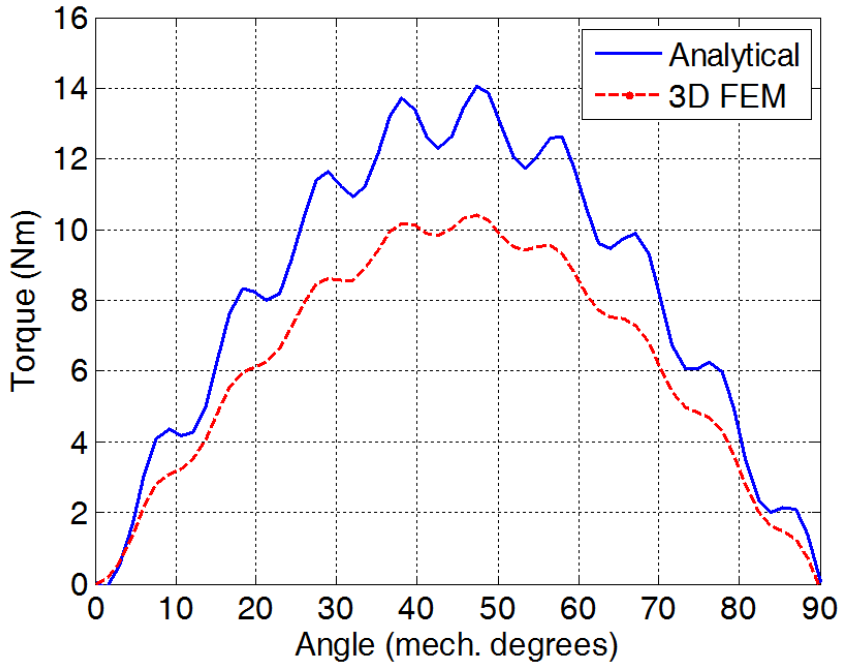

(a)

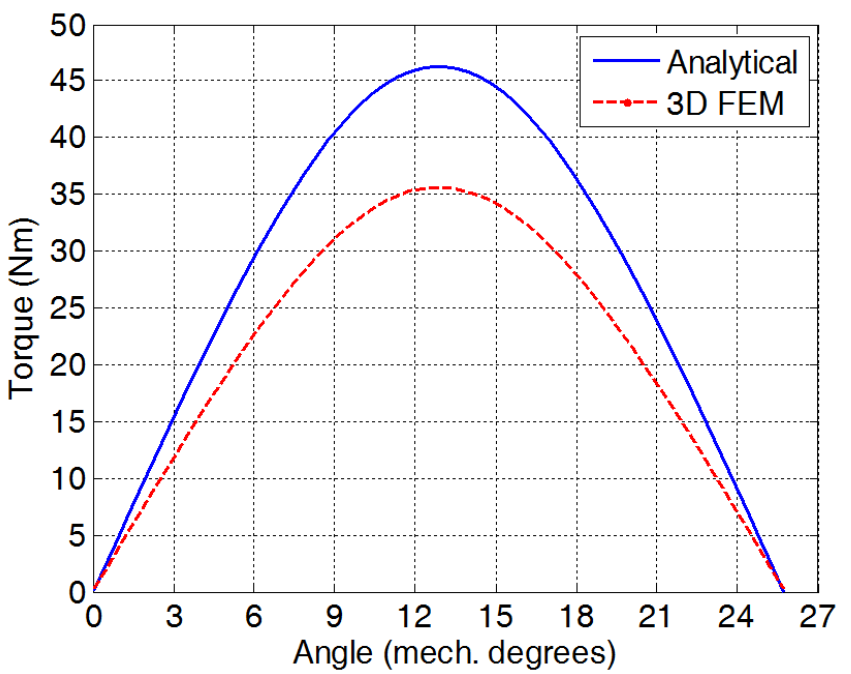

(b)

Fig. 8. (a) Torque exerted on the high-speed rotors versus $\delta_{h}\left(\delta_{l}=0 ; \theta_{0}=0\right)$; (b) Torque exerted on the low-speed rotor versus $\delta_{l}\left(\delta_{h}=0 ; \theta_{0}=0\right)$.

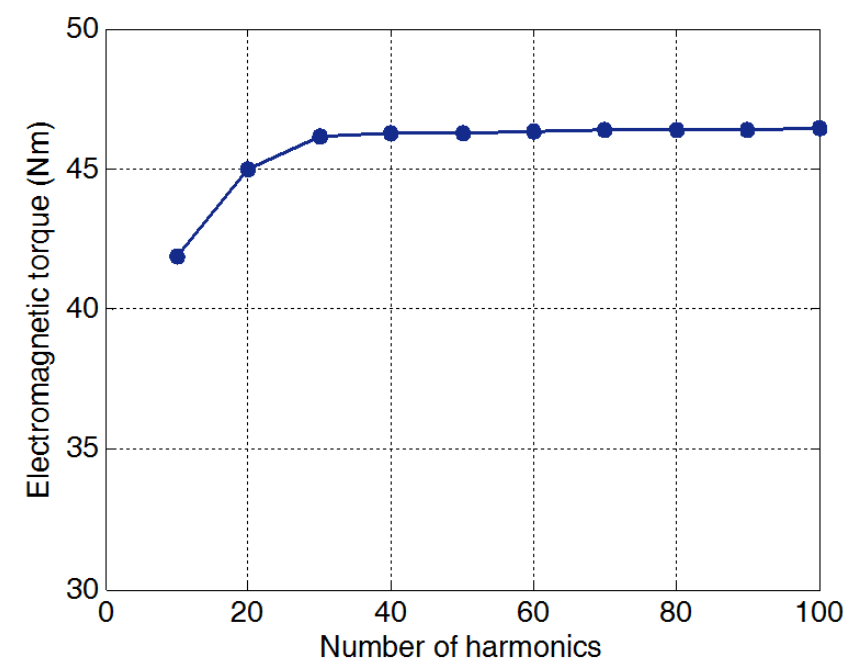

(a) 


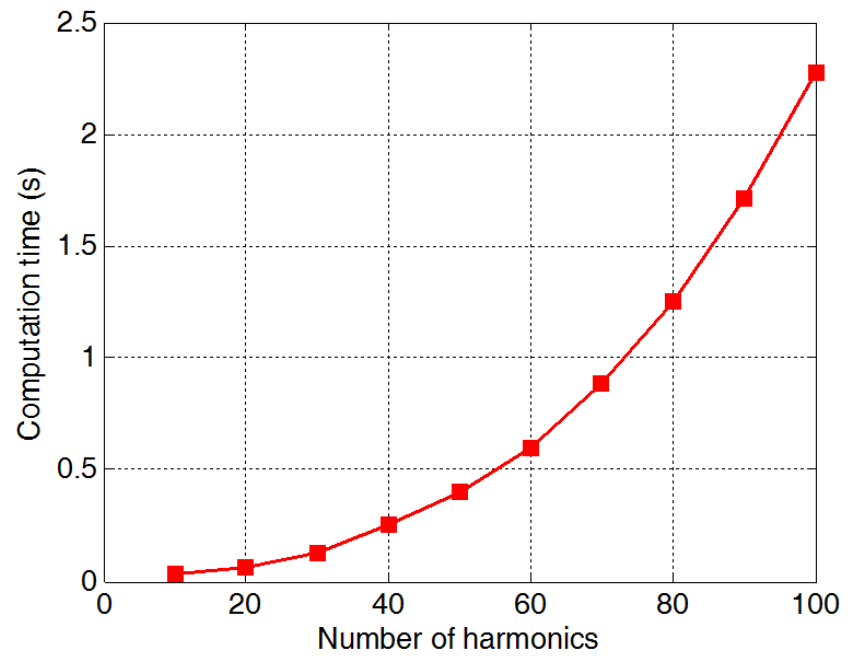

(b)

Fig. 9. Influence of the number of harmonic terms: (a) Maximum torque on the low speed rotor (b) computation time.

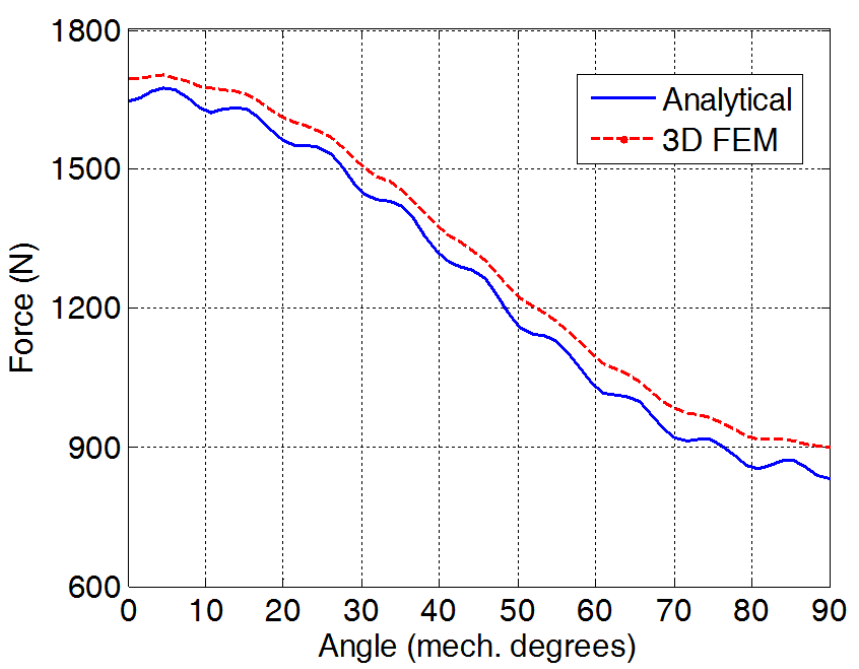

(a)

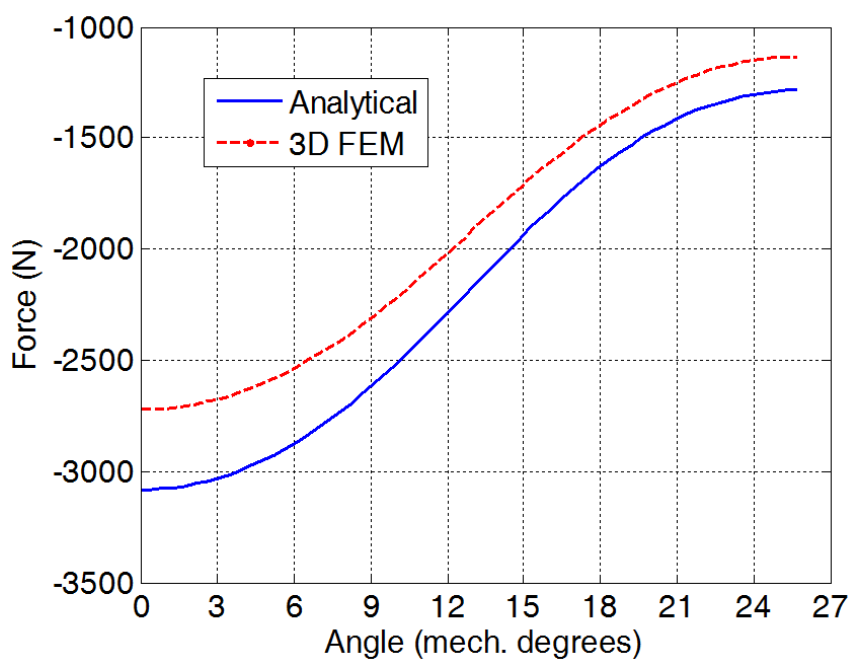

(b)

Fig. 10. a) Axial force acting on the high-speed rotors versus $\delta_{h}\left(\delta_{l}=0 ; \theta_{0}=\right.$ $0)$; (b) Axial force acting on the low-speed rotor versus $\delta_{l}$ for $\left(\delta_{h}=0 ; \theta_{0}=0\right)$.

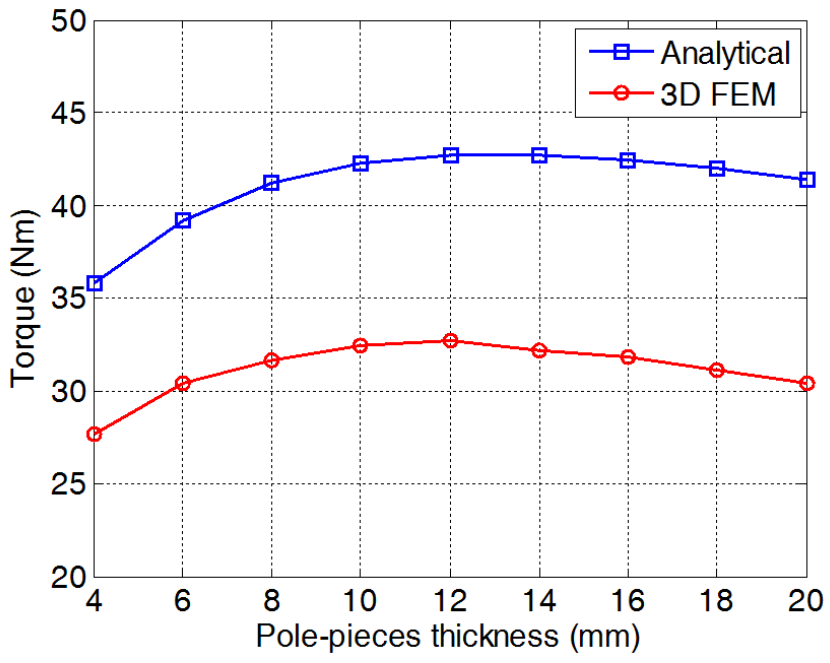

Fig. 11. Effect of the pole-pieces thickness on the torque value.

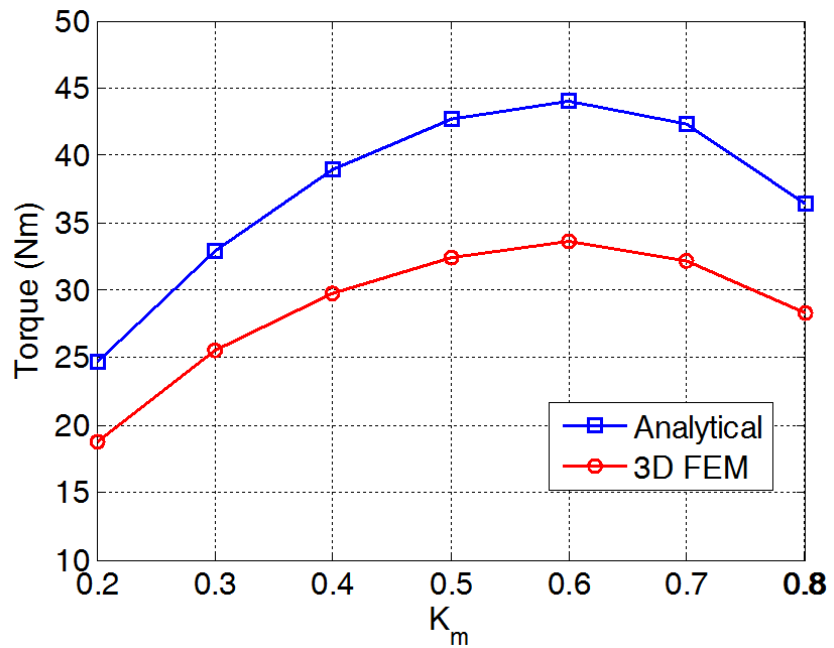

Fig. 12. Effect of the coefficient $\mathrm{K}_{\mathrm{m}}$ on the torque value.

\section{3-D Effects on the Flux Density Distribution}

To show the influence of the 3-D effects which are neglected in the proposed 2D analytical model, the flux density distributions (3D FE) along the radial coordinate $r$ (between $R_{1}$ and $R_{2}$ ) are plotted in Figs. 13 and 14 (the gear develops its pull out torque).

In each figure, the value of the flux density at the mean radius $r=R_{m}=0.05 \mathrm{~m}$ also corresponds to the one obtained using the $2 \mathrm{D}$ analytical model.

Fig. 13 shows the flux density distributions (axial and tangential components) along a radial coordinate situated behind a ferromagnetic pole-piece in the middle of the highspeed and the low-speed airgaps. As expected, the high permeability of the ferromagnetic pole-pieces leads to a very low tangential component (maximal value of $8 \mathrm{mT}$ in Fig. 13.a) so the axial component is predominant (Fig. 13.b). This axial component is relatively constant along $r$ and its value is not too far from the $2 \mathrm{D}$ one, except when approaching the external radius $R_{2}=0.07 \mathrm{~m}$. Indeed, between $r=0.065 \mathrm{~m}$ and $r=0.07 \mathrm{~m}$, the 
values of the flux density rapidly decreases from $-1 \mathrm{~T}$ to $-0.6 \mathrm{~T}$ in the low-speed airgap and from $-0.9 \mathrm{~T}$ to $-0.4 \mathrm{~T}$ in the highspeed airgap. This is mainly due to the fact that only nonmagnetic material (air) subsists when $r>R_{2}$.

Fig. 14 shows the flux density distributions (axial and tangential components) along a radial coordinate situated between two ferromagnetic pole-pieces in the middle of the high-speed and the low-speed airgaps. Unlike the preceding curves, the tangential components of the flux density take higher values and have large variations along $r$ (particularly in the low-speed air gap, Fig. 14.a).

Concerning the axial components (Fig. 14.b), their values are lower than the ones obtained in Fig.13.b, but remain practically constant along $r$. These values are close to the ones obtained with the 2D analytical model. A low decrease is noticed while approaching the external radius $R_{2}=0.07 \mathrm{~m}$.

Further investigations have been conducted at several orthoradial positions and lead to the same observations as above viz.:

1- along the radial coordinate, the tangential flux density evaluated by 3D FEM, has large variations which cannot be predicted by the 2D analytical model,

2- compared to the 3D FE computations, the 2D analytical model predicts with a good accuracy both the high and low speed airgap axial flux densities which only exhibit little variations along the radial coordinate

At least for the studied gear, observation no 1 constitutes the main reason of the relatively high difference between the torque predictions (see Fig.8) of the analytical 2D model and the exact 3D FE model. Indeed, the torque is produced by the interaction of the axial and tangential components of the flux density. In another hand, observation $\mathrm{n}^{\mathrm{o}} 2$ justifies the lower error in the evaluation of the axial forces (see Fig.10). Obviously, the presence of the ferromagnetic materials (polepieces and yokes) makes the axial force mainly dependent on the square of the axial flux density which is accurately predicted by the $2 \mathrm{D}$ analytical model.

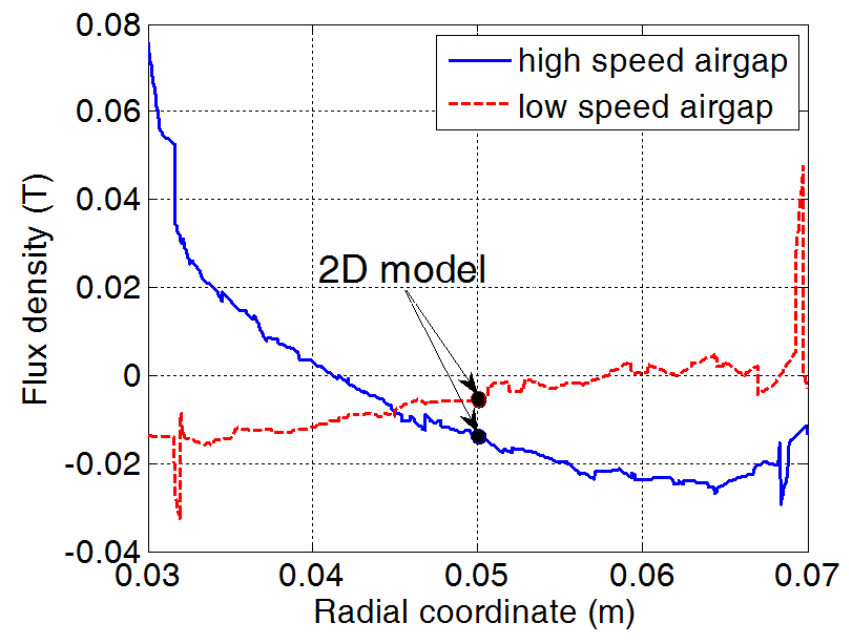

(a)

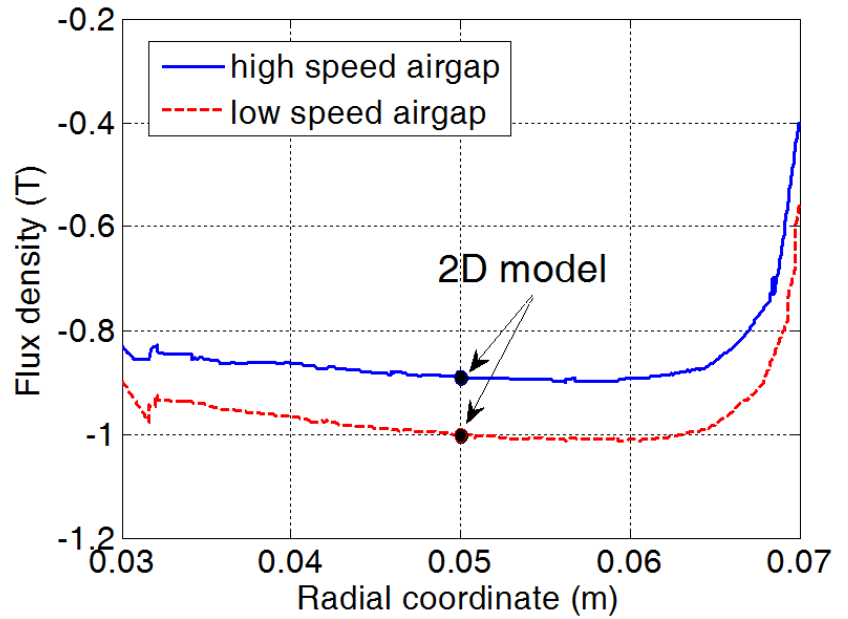

(b)

Fig. 13. Flux density distribution along a radial coordinate behind a ferromagnetic pole-piece a) tangential component b) axial component.

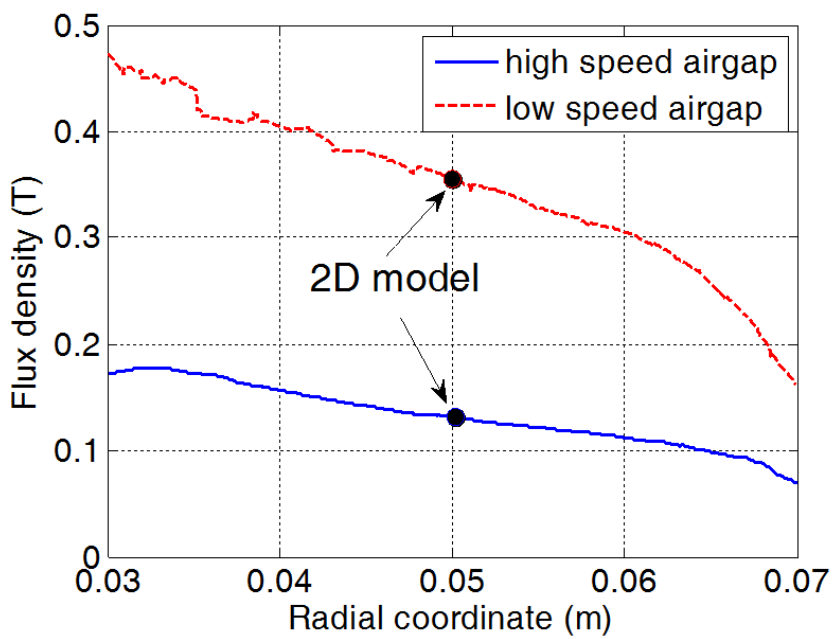

(a)

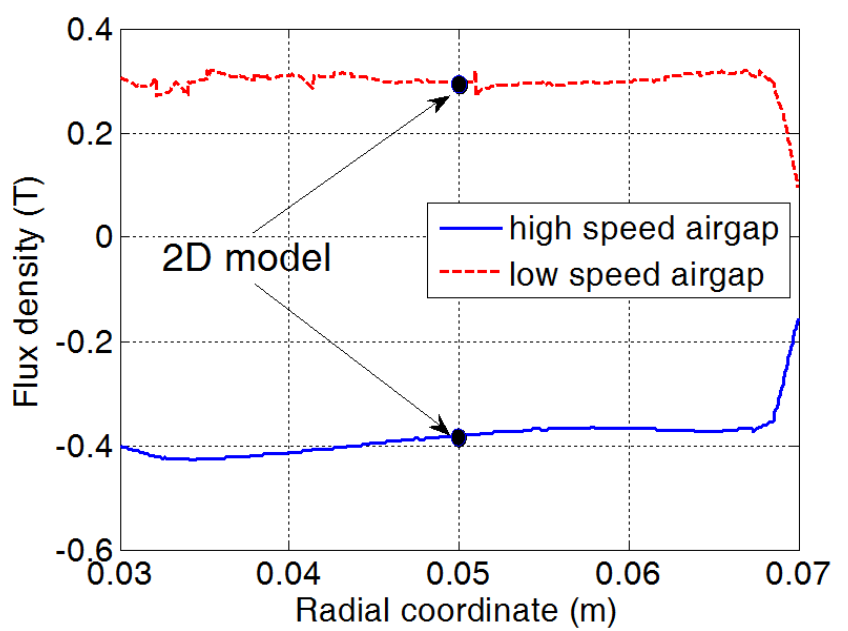

(b)

Fig. 14. Flux density distribution along a radial coordinate between ferromagnetic pole-pieces a) tangential component b) axial component. 


\section{CONCLUSION}

In this paper, we have developed a two-dimensional analytical model to predict the magnetic field distribution and the electromagnetic torque for axial-field magnetic gears. The proposed model is based on an exact 2D solution of the magnetic field at the mean radius. A special attention has been given to the solution of the magnetic field in a slot open on two sides.

We have shown that the flux density distribution in the airgap and slot regions (at the mean radius) computed with the analytical model is in close agreement with the one obtained using 3D FEM. The proposed 2D analytical model shows some lack of accuracy compared to $3 \mathrm{D}$ finite-element simulations for the torque prediction. It over-estimates the maximum torque value of about $30 \%$ (for the studied example). However, we have shown that the analytical model can be used to determine rapidly the optimal value of the ferromagnetic pole-pieces and magnets thickness when the other geometrical parameters are given. Indeed, the parametric studies show that the analytical and the 3D FE simulations follow the same trends in evaluating the electromagnetic torque.

The proposed analytical model has lower computational time than 3D FE simulations. Hence, it can be used as an effective tool for parametric studies or for the first step of the design optimization of axial-field magnetic gears.

\section{APPENDIX}

For the determination of the integration constants, we have to calculate integrals of the form

$$
\begin{gathered}
f(k, n, i)=\int_{\theta_{i}}^{\theta_{i}+\beta} \cos (n \theta) \cos \left(\frac{k \pi}{\beta}\left(\theta-\theta_{i}\right)\right) d \theta \\
g(k, n, i)=\int_{\theta_{i}}^{\theta_{i}+\beta} \sin (n \theta) \cos \left(\frac{k \pi}{\beta}\left(\theta-\theta_{i}\right)\right) d \theta \\
r(n, i)=\int_{\theta_{i}}^{\theta_{i}+\beta} \cos (n \theta) d \theta \\
s(n, i)=\int_{\theta_{i}}^{\theta_{i}+\beta} \sin (n \theta) d \theta
\end{gathered}
$$

The development of (A.1) and (A.2) gives the following functions that will be used in the expressions of the integration coefficients

$$
\begin{aligned}
& \text { - for } k \pi \neq n \beta \\
& \qquad \begin{aligned}
f(k, n, i) & =\frac{-n \beta^{2}\left((-1)^{k} \sin n\left(\beta+\theta_{i}\right)-\sin \left(n \theta_{i}\right)\right)}{k^{2} \pi^{2}-n^{2} \beta^{2}} \\
g(k, n, i) & =\frac{n \beta^{2}\left((-1)^{k} \cos n\left(\beta+\theta_{i}\right)-\cos \left(n \theta_{i}\right)\right)}{k^{2} \pi^{2}-n^{2} \beta^{2}}
\end{aligned}
\end{aligned}
$$

$$
\begin{aligned}
\text { - for } k \pi & =n \beta \\
f(k, n, i) & =\frac{\beta}{2}\left(\cos \left(n \theta_{i}\right)+\frac{1}{2 k \pi}\left(\sin n\left(\theta_{i}+2 \beta\right)-\sin \left(n \theta_{i}\right)\right)\right) \\
g(k, n, i) & =\frac{\beta}{2}\left(\sin \left(n \theta_{i}\right)-\frac{1}{2 k \pi}\left(\cos n\left(\theta_{i}+2 \beta\right)-\cos \left(n \theta_{i}\right)\right)\right)
\end{aligned}
$$

The development of (A.3) and (A.4) gives the following functions

$$
\begin{gathered}
r(n, i)=\frac{1}{n}\left(\sin \left(n \theta_{i}+n \beta\right)-\sin \left(n \theta_{i}\right)\right) \\
s(n, i)=\frac{1}{n}\left(-\cos \left(n \theta_{i}+n \beta\right)+\cos \left(n \theta_{i}\right)\right)
\end{gathered}
$$

The solution for the integration constants can be determined by solving a linear system of equations (Cramer's system) which can be written in matrix form as

$$
[M](X)=(S)
$$

where $(X)$ is the unknown vector defined as

$$
\begin{array}{r}
(X)=\left(\begin{array}{lllllllllll}
a_{n}^{I} & c_{n}^{I} & a_{n}^{I I} & b_{n}^{I I} & c_{n}^{I I} & d_{n}^{I I} & a_{0}^{i} & b_{0}^{i} & a_{k}^{i} & b_{k}^{i} & \ldots \\
& \ldots . & a_{n}^{I I I} & b_{n}^{I I I} & c_{n}^{I I I} & d_{n}^{I I I} & a_{n}^{I V} & c_{n}^{I V}
\end{array}\right)^{T}
\end{array}
$$

The matrix $[\boldsymbol{M}]$ and the source vector $(S)$ are developed in (A.54). In the following developments, $N$ represents the number of harmonic terms used in air-gap and magnet regions (regions $I, I I, I I I$ and $I V$ ) and $K$ the number of harmonic terms used in the slot regions (regions $i$ ). $Q$ is the number of slots. $I_{N}$ and $I_{Q}$ in (A.54) are respectively the identity matrices of dimension $\mathrm{N}$ and $Q$, and $I_{K Q}$ is the identity matrix of dimension $K \times Q$. The dimension of $(X)$ is then equal to $12 N+2 Q+2 K Q$.

To solve the linear system (A.11), a numerical matrix inversion is required for the calculation of the unknown vector. This can be done by using mathematical software (Matlab or Maple).

- Expressions of the constants $a_{n}^{I}, b_{n}^{I}$ : The development of (29) and (30) gives

$$
\begin{aligned}
& a_{n}^{I}+K_{n}^{h} \cos \left(n \delta_{h}\right)= \\
& a_{n}^{I I} \frac{R_{m}}{n} \frac{\operatorname{ch}\left(\frac{n}{R_{m}}\left(z_{1}-z_{2}\right)\right)}{\operatorname{sh}\left(\frac{n}{R_{m}}\left(z_{1}-z_{2}\right)\right)}+b_{n}^{I I} \frac{R_{m}}{n} \frac{1}{\operatorname{sh}\left(\frac{n}{R_{m}}\left(z_{2}-z_{1}\right)\right)} \\
& c_{n}^{I}+K_{n}^{h} \sin \left(n \delta_{h}\right)= \\
& c_{n}^{I I} \frac{R_{m}}{n} \frac{\operatorname{ch}\left(\frac{n}{R_{m}}\left(z_{1}-z_{2}\right)\right)}{\operatorname{sh}\left(\frac{n}{R_{m}}\left(z_{1}-z_{2}\right)\right)}+d_{n}^{I I} \frac{R_{m}}{n} \frac{1}{\operatorname{sh}\left(\frac{n}{R_{m}}\left(z_{2}-z_{1}\right)\right)}
\end{aligned}
$$


Equation (A.13) and (A.14) can be rewritten in vector and matrix form as indicated in lines 1 and 2 of (A.54) where

$$
\begin{aligned}
& \left(S_{1}\right)_{N}=K_{n}^{h} \cos \left(n \delta_{h}\right) \\
& \left(S_{2}\right)_{N}=K_{n}^{h} \sin \left(n \delta_{h}\right) \\
& \boldsymbol{M}_{N \times N}^{(1)}=\frac{R_{m}}{n} \frac{\operatorname{ch}\left(\frac{n}{R_{m}}\left(z_{1}-z_{2}\right)\right)}{\operatorname{sh}\left(\frac{n}{R_{m}}\left(z_{1}-z_{2}\right)\right)} \\
& \boldsymbol{M}_{N \times N}^{(2)}=\frac{R_{m}}{n} \frac{1}{\operatorname{sh}\left(\frac{n}{R_{m}}\left(z_{2}-z_{1}\right)\right)}
\end{aligned}
$$

where $N \times N$ (in $\boldsymbol{M}_{N \times N}^{(1)}$ ) indicates the matrix dimensions (rows $\times$ columns).

- Expressions of the coefficients $a_{n}^{I I}, b_{n}^{I I}, c_{n}^{I I}$ and $d_{n}^{I I}$ : The development of (39) to (42) gives

$$
\begin{aligned}
& a_{n}^{I I}=a_{n}^{I} \frac{n}{R_{m}} \operatorname{th}\left(\frac{n}{R_{m}} z_{1}\right) \\
& c_{n}^{I I}=c_{n}^{I} \frac{n}{R_{m}} \operatorname{th}\left(\frac{n}{R_{m}} z_{1}\right) \\
& b_{n}^{I I}=\sum_{i=1}^{Q} \frac{b_{0}^{i}}{\pi} r(n, i) \\
& +\sum_{i=1}^{Q} \sum_{k=1}^{K} a_{k}^{i} \frac{k}{\beta R_{m}} \frac{\operatorname{ch}\left(\frac{k \pi}{\beta R_{m}}\left(z_{2}-z_{3}\right)\right)}{\operatorname{sh}\left(\frac{k \pi}{\beta R_{m}}\left(z_{2}-z_{3}\right)\right)} f(k, n, i) \\
& +\sum_{i=1}^{Q} \sum_{k=1}^{K} b_{k}^{i} \frac{k}{\beta R_{m}} \frac{1}{\operatorname{sh}\left(\frac{k \pi}{\beta R_{m}}\left(z_{3}-z_{2}\right)\right)} f(k, n, i) \\
& d_{n}^{I I}=\sum_{i=1}^{Q} \frac{b_{0}^{i}}{\pi} s(n, i) \\
& +\sum_{i=1}^{Q} \sum_{k=1}^{K} a_{k}^{i} \frac{k}{\beta R_{m}} \frac{\operatorname{ch}\left(\frac{k \pi}{\beta R_{m}}\left(z_{2}-z_{3}\right)\right)}{\operatorname{sh}\left(\frac{k \pi}{\beta R_{m}}\left(z_{2}-z_{3}\right)\right)} g(k, n, i) \\
& +\sum_{i=1}^{Q} \sum_{k=1}^{K} b_{k}^{i} \frac{k}{\beta R_{m}} \frac{1}{\operatorname{sh}\left(\frac{k \pi}{\beta R_{m}}\left(z_{3}-z_{2}\right)\right)} g(k, n, i)
\end{aligned}
$$

(A.19) to (A.22) can be rewritten in vector and matrix form as indicated in lines 3, 4 and 5 of (A.54) where

$$
\boldsymbol{M}_{N \times N}^{(3)}=\frac{n}{R_{m}} \operatorname{th}\left(\frac{n}{R_{m}} z_{1}\right)
$$

$$
\begin{aligned}
& \boldsymbol{M}_{N \times Q}^{(4)}=\sum_{i=1}^{Q} \frac{r(n, i)}{\pi} \\
& \boldsymbol{M}_{N \times K Q}^{(5)}=\sum_{i=1}^{Q} \sum_{k=1}^{K} \frac{k}{\beta R_{m}} \frac{\operatorname{ch}\left(\frac{k \pi}{\beta R_{m}}\left(z_{2}-z_{3}\right)\right)}{\operatorname{sh}\left(\frac{k \pi}{\beta R_{m}}\left(z_{2}-z_{3}\right)\right)} f(k, n, i) \\
& \boldsymbol{M}_{N \times K Q}^{(6)}=\sum_{i=1}^{Q} \sum_{k=1}^{K} \frac{k}{\beta R_{m}} \frac{1}{\operatorname{sh}\left(\frac{k \pi}{\beta R_{m}}\left(z_{3}-z_{2}\right)\right)} f(k, n, i)
\end{aligned}
$$

$\boldsymbol{M}_{N \times Q}^{(7)}, \boldsymbol{M}_{N \times K Q}^{(8)}, \boldsymbol{M}_{N \times K Q}^{(9)}$ in line 6 of (A.54) have respectively the same form as $\boldsymbol{M}_{N \times Q}^{(4)}, \boldsymbol{M}_{N \times K Q}^{(5)}, \boldsymbol{M}_{N \times K Q}^{(6)}$ by replacing $r(n, i)$ by $s(n, i)$ and $f(k, n, i)$ by $g(k, n, i)$.

- Expression of the coefficients $a_{0}^{i}, b_{0}^{i}, a_{k}^{i}$ and $b_{k}^{i}$ : The treatment of (20) to (23) yields to the following linear relations

$$
\begin{aligned}
& a_{0}^{i}+b_{0}^{i} z_{2}= \\
& \sum_{n=1}^{N}\left(a_{n}^{I I} \frac{R_{m}}{n \beta} \frac{1}{s h\left(\frac{n}{R_{m}}\left(z_{1}-z_{2}\right)\right)}+b_{n}^{I I} \frac{R_{m}}{n \beta} \frac{\operatorname{ch}\left(\frac{n}{R_{m}}\left(z_{2}-z_{1}\right)\right)}{\operatorname{sh}\left(\frac{n}{R_{m}}\left(z_{2}-z_{1}\right)\right)} r(n, i)\right. \\
& +\sum_{n=1}^{N}\left(c_{n}^{I I} \frac{R_{m}}{n \beta} \frac{1}{s h\left(\frac{n}{R_{m}}\left(z_{1}-z_{2}\right)\right)}+d_{n}^{I I} \frac{R_{m}}{n \beta} \frac{\operatorname{ch}\left(\frac{n}{R_{m}}\left(z_{2}-z_{1}\right)\right)}{\operatorname{sh}\left(\frac{n}{R_{m}}\left(z_{2}-z_{1}\right)\right)} s(n, i)\right.
\end{aligned}
$$

which can be rewritten in vector and matrix form as indicated in line 7 of (A.54) where

$$
\begin{gathered}
\boldsymbol{M}_{Q \times N}^{(10)}=\sum_{n=1}^{N} \frac{R_{m}}{n \beta} \frac{1}{\operatorname{sh}\left(\frac{n}{R_{m}}\left(z_{1}-z_{2}\right)\right)} r(n, i) \\
\boldsymbol{M}_{Q \times N}^{(11)}=\sum_{n=1}^{N} \frac{R_{m}}{n \beta} \frac{\operatorname{ch}\left(\frac{n}{R_{m}}\left(z_{2}-z_{1}\right)\right)}{\operatorname{sh}\left(\frac{n}{R_{m}}\left(z_{2}-z_{1}\right)\right)} r(n, i)
\end{gathered}
$$

Matrices $\boldsymbol{M}_{Q \times N}^{(12)}, \boldsymbol{M}_{Q \times N}^{(13)}$ in line 7 of (A.54) have respectively the same form as $\boldsymbol{M}_{Q \times N}^{(10)}$ and $\boldsymbol{M}_{Q \times N}^{(11)}$ by replacing $r(n, i)$ by $s(n, i)$. 
$a_{0}^{i}+b_{0}^{i} z_{3}=$

$\sum_{n=1}^{N}\left(a_{n}^{I I I} \frac{R_{m}}{n \beta} \frac{\operatorname{ch}\left(\frac{n}{R_{m}}\left(z_{3}-z_{4}\right)\right)}{\operatorname{sh}\left(\frac{n}{R_{m}}\left(z_{3}-z_{4}\right)\right)}+b_{n}^{I I I} \frac{R_{m}}{n \beta} \frac{1}{\operatorname{sh}\left(\frac{n}{R_{m}}\left(z_{4}-z_{3}\right)\right)}\right) r(n, i)$

$+\sum_{n=1}^{N}\left(c_{n}^{I I I} \frac{R_{m}}{n \beta} \frac{\operatorname{ch}\left(\frac{n}{R_{m}}\left(z_{3}-z_{4}\right)\right)}{\operatorname{sh}\left(\frac{n}{R_{m}}\left(z_{3}-z_{4}\right)\right)}+d_{n}^{I I I} \frac{R_{m}}{n \beta} \frac{1}{\operatorname{sh}\left(\frac{n}{R_{m}}\left(z_{4}-z_{3}\right)\right)}\right) s(n, i)$

(A.30)

which can be rewritten in vector and matrix form as indicated in line 8 of (A.54) where

$$
\begin{gathered}
\boldsymbol{M}_{Q \times N}^{(14)}=\sum_{n=1}^{N} \frac{R_{m}}{n \beta} \frac{\operatorname{ch}\left(\frac{n}{R_{m}}\left(z_{3}-z_{4}\right)\right)}{\operatorname{sh}\left(\frac{n}{R_{m}}\left(z_{3}-z_{4}\right)\right)} r(n, i) \\
\boldsymbol{M}_{Q \times N}^{(15)}=\sum_{n=1}^{N} \frac{R_{m}}{n \beta} \frac{1}{\operatorname{sh}\left(\frac{n}{R_{m}}\left(z_{4}-z_{3}\right)\right)} r(n, i)
\end{gathered}
$$

$\boldsymbol{M}_{Q \times N}^{(16)}, \boldsymbol{M}_{Q \times N}^{(17)}$ in line 8 of (A.54) have respectively the same form as $\boldsymbol{M}_{Q \times N}^{(14)}$ and $\boldsymbol{M}_{Q \times N}^{(15)}$ by replacing $r(n, i)$ by $s(n, i)$.

$$
\begin{aligned}
& a_{k}^{i}= \\
& \sum_{n=1}^{N}\left(a_{n}^{I I} \frac{2 R_{m}}{n \beta} \frac{1}{\operatorname{sh}\left(\frac{n}{R_{m}}\left(z_{1}-z_{2}\right)\right)}+b_{n}^{I I} \frac{2 R_{m}}{n \beta} \frac{\operatorname{ch}\left(\frac{n}{R_{m}}\left(z_{2}-z_{1}\right)\right)}{\operatorname{sh}\left(\frac{n}{R_{m}}\left(z_{2}-z_{1}\right)\right)}\right) f(k, n, i) \\
& +\sum_{n=1}^{N}\left(c_{n}^{I I} \frac{2 R_{m}}{n \beta} \frac{1}{\operatorname{sh}\left(\frac{n}{R_{m}}\left(z_{1}-z_{2}\right)\right)}+d_{n}^{I I} \frac{2 R_{m}}{n \beta} \frac{\operatorname{ch}\left(\frac{n}{R_{m}}\left(z_{2}-z_{1}\right)\right)}{\operatorname{sh}\left(\frac{n}{R_{m}}\left(z_{2}-z_{1}\right)\right)} g(k, n, i)\right.
\end{aligned}
$$

which can be rewritten in vector and matrix form as indicated in line 9 of (A.54) as

$$
\begin{gathered}
\boldsymbol{M}_{K Q \times N}^{(18)}=\sum_{n=1}^{N} \frac{2 R_{m}}{n \beta} \frac{1}{\operatorname{sh}\left(\frac{n}{R_{m}}\left(z_{1}-z_{2}\right)\right)} f(k, n, i) \\
\boldsymbol{M}_{K Q \times N}^{(19)}=\sum_{n=1}^{N} \frac{2 R_{m}}{n \beta} \frac{\operatorname{ch}\left(\frac{n}{R_{m}}\left(z_{2}-z_{1}\right)\right)}{\operatorname{sh}\left(\frac{n}{R_{m}}\left(z_{2}-z_{1}\right)\right)} f(k, n, i)
\end{gathered}
$$

$\boldsymbol{M}_{K Q \times N}^{(20)}$ and $\boldsymbol{M}_{K Q \times N}^{(21)}$ in line 9 of (A.54) have respectively the same form as $\boldsymbol{M}_{K Q \times N}^{(18)}$ and $\boldsymbol{M}_{K Q \times N}^{(19)}$ by replacing $f(\mathrm{k}, n, i)$ by $g(\mathrm{k}, n, i)$. It is worth noting that the vector $\left(a_{k}^{i}\right)_{K Q}$ in line 9 of (A.54) is of dimension $K \times Q$ and takes into account the $Q$ slots.

$$
\begin{aligned}
& b_{k}^{i}= \\
& \sum_{n=1}^{N}\left(a_{n}^{I I I} \frac{2 R_{m}}{n \beta} \frac{\operatorname{ch}\left(\frac{n}{R_{m}}\left(z_{3}-z_{4}\right)\right)}{\operatorname{sh}\left(\frac{n}{R_{m}}\left(z_{3}-z_{4}\right)\right)}+b_{n}^{I I I} \frac{2 R_{m}}{n \beta} \frac{1}{\operatorname{sh}\left(\frac{n}{R_{m}}\left(z_{4}-z_{3}\right)\right)}\right) f(k, n, i) \\
& +\sum_{n=1}^{N}\left(c_{n}^{I I I} \frac{2 R_{m}}{n \beta} \frac{\operatorname{ch}\left(\frac{n}{R_{m}}\left(z_{3}-z_{4}\right)\right)}{\operatorname{sh}\left(\frac{n}{R_{m}}\left(z_{3}-z_{4}\right)\right)}+d_{n}^{I I I} \frac{2 R_{m}}{n \beta} \frac{1}{\operatorname{sh}\left(\frac{n}{R_{m}}\left(z_{4}-z_{3}\right)\right)}\right) g(k, n, i)
\end{aligned}
$$

which can be rewritten in vector and matrix form as indicated in line 10 of (A.54) as

$$
\begin{gathered}
\boldsymbol{M}_{K Q \times N}^{(22)}=\sum_{n=1}^{N} \frac{2 R_{m}}{n \beta} \frac{\operatorname{ch}\left(\frac{n}{R_{m}}\left(z_{3}-z_{4}\right)\right)}{\operatorname{sh}\left(\frac{n}{R_{m}}\left(z_{3}-z_{4}\right)\right)} f(k, n, i) \\
\boldsymbol{M}_{K Q \times N}^{(23)}=\sum_{n=1}^{N} \frac{2 R_{m}}{n \beta} \frac{1}{\operatorname{sh}\left(\frac{n}{R_{m}}\left(z_{4}-z_{3}\right)\right)} f(k, n, i)
\end{gathered}
$$

$\boldsymbol{M}_{K Q \times N}^{(24)}$ and $\boldsymbol{M}_{K Q \times N}^{(25)}$ in line 10 of (A.54) have respectively the same form as $\boldsymbol{M}_{K Q \times N}^{(22)}$ and $\boldsymbol{M}_{K Q \times N}^{(23)}$ by replacing $f(\mathrm{k}, n, i)$ by $g(\mathrm{k}, n, i)$.

- Expressions of the coefficients $a_{n}^{I I I}, b_{n}^{I I I}, c_{n}^{I I I}$ and $d_{n}^{I I I}$ : The development of (47) to (50) gives

$$
\begin{aligned}
& b_{n}^{I I I}=a_{n}^{I V} \frac{n}{R_{m}} \operatorname{th}\left(\frac{n}{R_{m}}\left(z_{4}-z_{5}\right)\right) \\
& d_{n}^{I I I}=c_{n}^{I V} \frac{n}{R_{m}} \operatorname{th}\left(\frac{n}{R_{m}}\left(z_{4}-z_{5}\right)\right) \\
& a_{n}^{I I I}=\sum_{i=1}^{Q} \frac{b_{0}^{i}}{\pi} r(n, i) \\
& +\sum_{i=1}^{Q} \sum_{k=1}^{K} a_{k}^{i} \frac{k}{\beta R_{m}} \frac{1}{\operatorname{sh}\left(\frac{k \pi}{\beta R_{m}}\left(z_{2}-z_{3}\right)\right)} f(k, n, i) \\
& +\sum_{i=1}^{Q} \sum_{k=1}^{K} b_{k}^{i} \frac{k}{\beta R_{m}} \frac{\operatorname{ch}\left(\frac{k \pi}{\beta R_{m}}\left(z_{3}-z_{2}\right)\right)}{\operatorname{sh}\left(\frac{k \pi}{\beta R_{m}}\left(z_{3}-z_{2}\right)\right)} f(k, n, i)
\end{aligned}
$$




$$
\begin{aligned}
& c_{n}^{I I I}=\sum_{i=1}^{Q} \frac{b_{0}^{i}}{\pi} s(n, i) \\
& +\sum_{i=1}^{Q} \sum_{k=1}^{K} a_{k}^{i} \frac{k}{\beta R_{m}} \frac{1}{\operatorname{sh}\left(\frac{k \pi}{\beta R_{m}}\left(z_{2}-z_{3}\right)\right)} g(k, n, i) \\
& +\sum_{i=1}^{Q} \sum_{k=1}^{K} b_{k}^{i} \frac{k}{\beta R_{m}} \frac{\operatorname{ch}\left(\frac{k \pi}{\beta R_{m}}\left(z_{3}-z_{2}\right)\right)}{\operatorname{sh}\left(\frac{k \pi}{\beta R_{m}}\left(z_{3}-z_{2}\right)\right)} g(k, n, i)
\end{aligned}
$$

(A.39) to (A.42) can be rewritten in vector and matrix form as indicated in lines $11,12,13$ and 14 of (A.54) where

$$
\begin{aligned}
& \boldsymbol{M}_{N \times Q}^{(26)}=\sum_{i=1}^{Q} \frac{r(n, i)}{\pi} \\
& \boldsymbol{M}_{N \times K Q}^{(27)}=\sum_{i=1}^{Q} \sum_{k=1}^{K} \frac{k}{\beta R_{m}} \frac{1}{\operatorname{sh}\left(\frac{k \pi}{\beta R_{m}}\left(z_{2}-z_{3}\right)\right)} f(k, n, i) \\
& \boldsymbol{M}_{N \times K Q}^{(28)}=\sum_{i=1}^{Q} \sum_{k=1}^{K} \frac{k}{\beta R_{m}} \frac{\operatorname{ch}\left(\frac{k \pi}{\beta R_{m}}\left(z_{3}-z_{2}\right)\right)}{\operatorname{sh}\left(\frac{k \pi}{\beta R_{m}}\left(z_{3}-z_{2}\right)\right)} f(k, n, i) \\
& \boldsymbol{M}_{N \times N}^{(29)}=\frac{n}{R_{m}} \operatorname{th}\left(\frac{n}{R_{m}}\left(z_{4}-z_{5}\right)\right)
\end{aligned}
$$

$\boldsymbol{M}_{N \times Q}^{(30)}, \boldsymbol{M}_{N \times K Q}^{(31)}, \boldsymbol{M}_{N \times K Q}^{(32)}$ in line 13 of (A.54) have respectively the same form as $\boldsymbol{M}_{N \times Q}^{(26)}, \boldsymbol{M}_{N \times K Q}^{(27)}, \boldsymbol{M}_{N \times K Q}^{(28)}$ by replacing $r(n, i)$ by $s(n, i)$ and $f(k, n, i)$ by $g(k, n, i)$.

- Expressions of the coefficients $a_{n}^{I V}, b_{n}^{I V}$ : The development of (33) and (34) gives

$$
\begin{aligned}
& a_{n}^{I V}+K_{n}^{l} \cos \left(n \delta_{l}\right)= \\
& a_{n}^{I I I} \frac{R_{m}}{n} \frac{1}{\operatorname{sh}\left(\frac{n}{R_{m}}\left(z_{3}-z_{4}\right)\right)}+b_{n}^{I I I} \frac{R_{m}}{n} \frac{\operatorname{ch}\left(\frac{n}{R_{m}}\left(z_{4}-z_{3}\right)\right)}{\operatorname{sh}\left(\frac{n}{R_{m}}\left(z_{4}-z_{3}\right)\right)} \\
& c_{n}^{I V}+K_{n}^{l} \sin \left(n \delta_{l}\right)= \\
& c_{n}^{I I I} \frac{R_{m}}{n} \frac{1}{\operatorname{sh}\left(\frac{n}{R_{m}}\left(z_{3}-z_{4}\right)\right)}+d_{n}^{I I I} \frac{R_{m}}{n} \frac{\operatorname{ch}\left(\frac{n}{R_{m}}\left(z_{4}-z_{3}\right)\right)}{\operatorname{sh}\left(\frac{n}{R_{m}}\left(z_{4}-z_{3}\right)\right)}
\end{aligned}
$$

Equation (A.47) and (A.48) can be rewritten in vector and matrix form as indicated lines 15 and 16 of (A.54) where

$$
\begin{aligned}
\left(S_{15}\right)_{N} & =K_{n}^{l} \cos \left(n \delta_{l}\right) \\
\left(S_{16}\right)_{N} & =K_{n}^{l} \sin \left(n \delta_{l}\right) \\
\boldsymbol{M}_{N \times N}^{(33)} & =\frac{R_{m}}{n} \frac{1}{\operatorname{sh}\left(\frac{n}{R_{m}}\left(z_{3}-z_{4}\right)\right)} \\
\boldsymbol{M}_{N \times N}^{(34)} & =\frac{R_{m}}{n} \frac{\operatorname{ch}\left(\frac{n}{R_{m}}\left(z_{4}-z_{3}\right)\right)}{\operatorname{sh}\left(\frac{n}{R_{m}}\left(z_{4}-z_{3}\right)\right)}
\end{aligned}
$$

- Moreover, we must add to these equations the supplementary constraint given by (44). The last line in (A.54) corresponds to (44).

- Expression of the coefficients $W_{n}, X_{n}, Y_{n}$ and $Z_{n}$ given in the torque and axial force equations (53) and (54)

$$
\begin{aligned}
& W_{n}=a_{n}^{I I} \frac{\operatorname{sh}\left(\frac{n}{R_{m}}\left(\zeta-z_{2}\right)\right)}{\operatorname{sh}\left(\frac{n}{R_{m}}\left(z_{1}-z_{2}\right)\right)}+b_{n}^{I I} \frac{\operatorname{sh}\left(\frac{n}{R_{m}}\left(\zeta-z_{1}\right)\right)}{\operatorname{sh}\left(\frac{n}{R_{m}}\left(z_{2}-z_{1}\right)\right)} \\
& Y_{n}=c_{n}^{I I} \frac{\operatorname{sh}\left(\frac{n}{R_{m}}\left(\zeta-z_{2}\right)\right)}{\operatorname{sh}\left(\frac{n}{R_{m}}\left(z_{1}-z_{2}\right)\right)}+d_{n}^{I I} \frac{\operatorname{sh}\left(\frac{n}{R_{m}}\left(\zeta-z_{1}\right)\right)}{\operatorname{sh}\left(\frac{n}{R_{m}}\left(z_{2}-z_{1}\right)\right)} \\
& Z_{n}=a_{n}^{I I} \frac{\operatorname{ch}\left(\frac{n}{R_{m}}\left(\zeta-z_{2}\right)\right)}{\operatorname{sh}\left(\frac{n}{R_{m}}\left(z_{1}-z_{2}\right)\right)}+b_{n}^{I I} \frac{\operatorname{ch}\left(\frac{n}{R_{m}}\left(\zeta-z_{1}\right)\right)}{\operatorname{sh}\left(\frac{n}{R_{m}}\left(z_{2}-z_{1}\right)\right)} \\
& X_{n}=-c_{n}^{I I} \frac{\operatorname{ch}\left(\frac{n}{R_{m}}\left(\zeta-z_{2}\right)\right)}{\operatorname{sh}\left(\frac{n}{R_{m}}\left(z_{1}-z_{2}\right)\right)}-d_{n}^{I I} \frac{\operatorname{ch}\left(\frac{n}{R_{m}}\left(\zeta-z_{1}\right)\right)}{\operatorname{sh}\left(\frac{n}{R_{m}}\left(z_{2}-z_{1}\right)\right)}
\end{aligned}
$$

\section{REFERENCES}

[1] K. Atallah and D. Howe, "A novel high-performance magnetic gear," IEEE Trans. Magn., vol. 37, no. 4, pp. 2844-2846, Jul. 2001.

[2] K. Atallah, S. Calverley and D. Howe, "Design, analysis and realization of a high-performance magnetic gear," IEE Proc. Electric Power Appl., vol. 151 , no. 2, pp. 135-143, 2004.

[3] S. Mezani, K. Atallah, and D. Howe, "A high-performance axial-field magnetic gear,” J. Appl. Phys., vol. 99, 08R303, 2006.

[4] P. O. Rasmussen, T. O. Andersen, F. T. Jorgensen, and O. Nielsen, "Development of a high-performance magnetic gear," IEEE Trans. Ind. Appl., vol. 41, no. 3, pp. 764-770, May/Jun. 2005.

[5] L. Jian, K. T. Chau, and J. Z. Jiang, "A magnetic-geared outer-rotor permanent-magnet brushless machine for wind power generation," IEEE Trans. Ind. Appl., vol. 35, no. 3, pp. 954-92, May/Jun. 2009.

[6] E. Gouda, S. Mezani, L. Baghli, and A. Rezzoug, "Comparative study between mechanical and magnetic planetary gears," IEEE Trans. Magn., vol. 47, no. 2, pp. 439-450, Feb. 2011.

[7] R. C. Holehouse, K. Atallah, and J. Wang, "Design and realization of a linear magnetic gear," IEEE Trans. Magn., vol. 47, no. 10, pp. 41714174, Oct. 2011.

[8] N. W. Franck, and H. A. Toliyat, "Analysis of the concentric planetary magnetic gear with strengthened stator and interior permanent magnet 
inner rotor," IEEE Trans. Ind. Appl., vol. 47, no. 4, pp. 1652-1660, Jul./Aug.. 2011.

[9] Z. Q. Zhu and D. Howe, "Instantaneous magnetic-field distribution in brushless permanent-magnet dc motor, part III: Effect of slotting," IEEE Trans. Magn., vol. 29, no. 1, pp. 143-151, Jan. 1993.

[10] B. Ackermann and R. Sottek, "Analytical modeling of the cogging torque in permanent magnet motors," Elect. Eng., vol. 78, no. 2, pp. 117-125, Mar. 1994.

[11] Z. J. Liu, and J. T. Li, "Accurate prediction of magnetic field and magnetic forces in permanent magnet motor using an analytical solution," IEEE Trans. Energy. Convers., vol. 23, no. 3, pp. 717-726, Sept. 2008.

[12] F. Dubas, and C. Espanet "Analytical solution of the magnetic field in permanent-magnet motors taking into account slotting effect: no-load vector potential and flux density calculation," IEEE Trans. Magn., vol. 45, no. 5, pp. 2097-21092, May 2009.

[13] Z. Q. Zhu, L. J. Wu, and Z.P. Xia, "An accurate subdomain model for magnetic field computation in slotted surface-mounted permanent magnet machines," IEEE Trans. Magn., vol. 46, no. 4, pp. 1100-1115, Apr. 2010.

[14] A. Bellara, Y. Amara, G. Barakat, and B. Dakyo, "Two-dimensional exact analytical solution of armature reaction field in slotted surface mounted PM radial flux synchronous machines," IEEE Trans. Magn., vol. 45, no. 10, pp. 4534-4538, Oct. 2009.

[15] Z. Q. Zhu, D. Howe, and Z. P. Xia, "Prediction of open-circuit airgap field distribution in brushless machines having an inset permanent magnet rotor topology," IEEE Trans. Magn., vol. 30, no. 1, pp. 98-107, Jan. 1994.

[16] L. Jian, K. T. Chau, Y. Gong, C. Yu, and W. Li, "Analytical calculation of magnetic field in surface-inset permanent magnet motors, " IEEE Trans. Magn., vol. 45, no. 10, pp. 4688-4691, Oct. 2009.

[17] L. Jian, and K. T. Chau, "Analytical calculation of magnetic field distribution in coaxial magnetic gears," Progress In Electromagnetics Research, vol. 92, no. 7, pp. 1-16, 2009.
[18] T. Lubin, S. Mezani, and A. Rezzoug, "Analytical computation of the magnetic field distribution in a magnetic gear," IEEE Trans. Magn., vol. 46, no. 7, pp. 2611-2621, Jul. 2010

[19] L. J. Wu, Z. Q. Zhu, D. Staton, M. Popescu, and D. Hawkins, “An improved subdomain model for predicting magnetic field of surfacemounted permanent magnet machines accounting for tooth-tips , "IEEE Trans. Magn., vol. 47, no. 6, pp. 1693-1704, Jun. 2011.

[20] T. Lubin, S. Mezani, and A. Rezzoug, "2-D Exact analytical model for surface-mounted permanent-magnet motors with semi-closed slots," IEEE Trans. Magn., vol. 47, no. 2, pp. 479-492, Feb. 2011.

[21] H. Tiegna, A. Bellara, Y. Amara, and G. Barakat, "Analytical modeling of the open-circuit magnetic field in axial flux permanent-magnet machines with semi-closed slots," IEEE Trans. Magn., vol. 48, no. 3, pp. 1212-1226, Mar. 2012.

[22] B. L. J. Gysen, K. J. Meessen, J. J. H. Paulides, and E. A. Lomonova, "General formulation of the electromagnetic field distribution in machines and devices using Fourier analysis," IEEE Trans. Magn., vol. 46, no. 1, pp. 39-52, Jan. 2010.

[23] T. Lubin, S. Mezani, and A. Rezzoug, "Simple analytical expressions for the force and torque of axial magnetic couplings," IEEE Trans. Energy. Convers., vol. 27, no. 2, pp. 536-546, Jun. 2012.

[24] A. Abdel-Khalik, S. Ahmed, A. Massoud and A. Elserougi, "Magnetic gearbox with an electric power output port and fixed speed ratio for wind energy applications", Proc. 20th Int. Conf. Elect. Mach. (ICEM), pp. 702-708, Sep. 2012.

[25] K. J. Meessen, B. L. J. Gysen, J. J. H. Paulides, and E. A. Lomonova, "General formulation of fringing fields in 3-D cylindrical structures using Fourier analysis," IEEE Trans. Magn., vol. 48, no. 8, pp. 23072323, Aug. 2012.

[26] S. J. Farlow, Partial Differential Equations for Scientists and Engineers. Dover publications, New York, 414 pp, 1993. 
Linear system of equations (Cramer's system) for the computation of the unknown coefficients

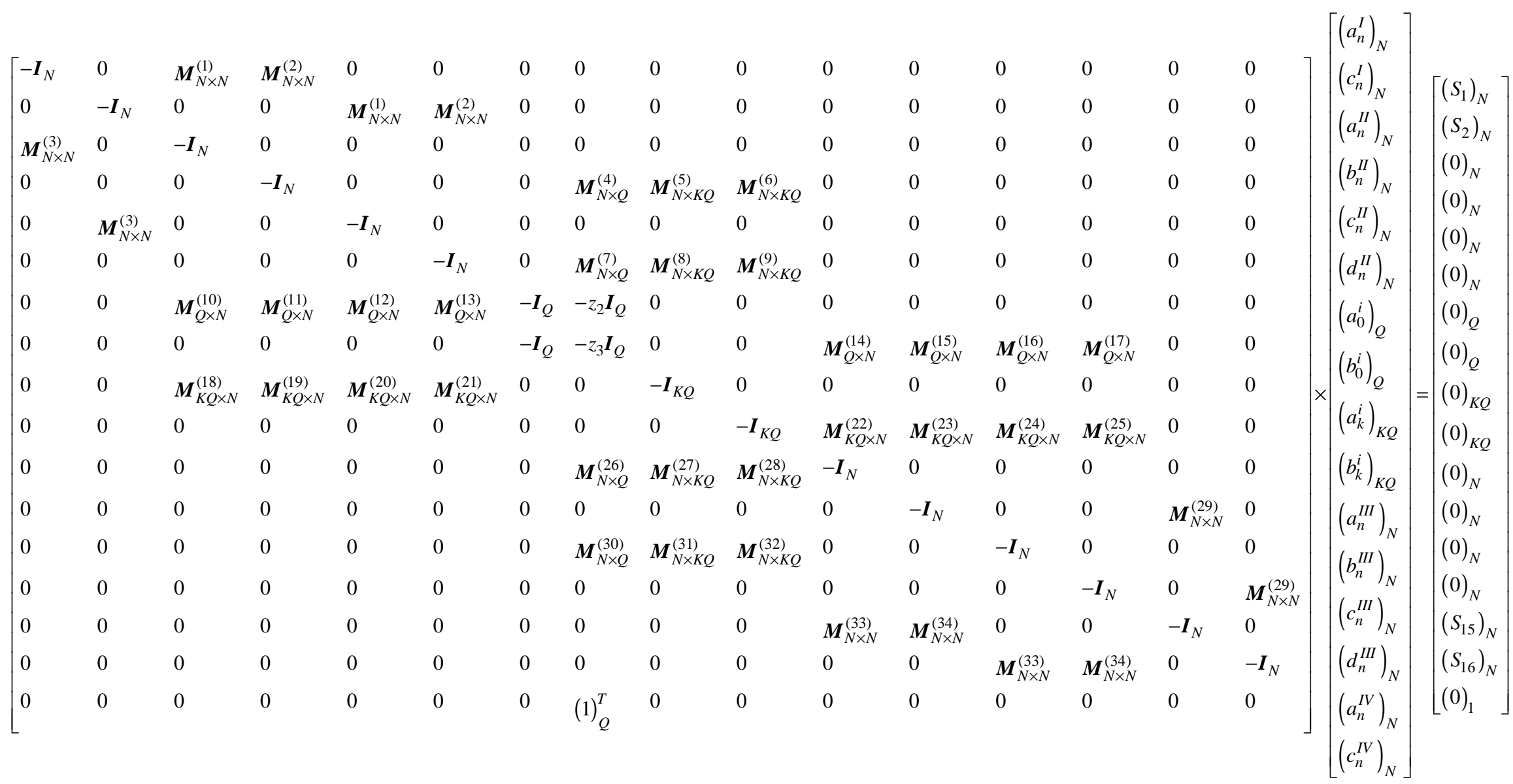

